\title{
Dark Matter Signatures in the Anisotropic Radio Sky
}

\author{
Le Zhang†, Günter Sigl† \\ $\dagger$ †I. Institut für theoretische Physik, Universität Hamburg, Luruper Chaussee 149, \\ D-22761 Hamburg, Germany
}

\begin{abstract}
We calculate intensity and angular power spectrum of the cosmological background of synchrotron emission from cold dark matter annihilations into electron positron pairs. We compare this background with intensity and anisotropy of astrophysical and cosmological radio backgrounds, such as from normal galaxies, radiogalaxies, galaxy cluster accretion shocks, the cosmic microwave background and with Galactic foregrounds. Under modest assumptions for the dark matter clustering we find that around $2 \mathrm{GHz}$ average intensity and fluctuations of the radio background at sub-degree scales allows to probe dark matter masses $\gtrsim 100 \mathrm{GeV}$ and annihilation cross sections not far from the natural values $\langle\sigma v\rangle \sim 3 \times 10^{-26} \mathrm{~cm}^{3} \mathrm{~s}^{-1}$ required to reproduce the correct relic density of thermal dark matter. The angular power spectrum of the signal from dark matter annihilation tends to be flatter than that from astrophysical radio backgrounds. Furthermore, radio source counts have comparable constraining power. Such signatures are interesting especially for future radio detectors such as SKA.
\end{abstract}

PACS numbers: 95.35.+d, 95.85.Bh, 98.70.Vc 


\section{Introduction}

Whereas it is known from cosmological observations that cold dark matter represents a fraction $\Omega_{m} \simeq 0.233$ of the total present energy density of our Universe [1, 2], its nature is still elusive. Dark matter can not only be detected directly in dedicated experiments searching for nuclear recoils from the scattering of dark matter particles, or produced in particle accelerators such as the LHC, but can also reveal its existence indirectly [3]: Although, apart from dilution from cosmic expansion, the density of dark matter does not change significantly after self-annihilations freeze out in the early Universe, residual self-annihilation can give rise to significant fluxes of $\gamma$-rays, electrons, positrons, neutrinos, and even some antimatter such as anti-protons and positrons, especially in regions with large dark matter densities. The energies of the secondary particles can reach up to the dark matter particle mass which can be of order a few hundred GeV. Secondary electrons and positrons can annihilate and give rise to a 511 $\mathrm{keV}$ line emission, and they emit synchrotron radiation in the magnetic fields of galaxies which can be detected in the radio band. Therefore, cosmic and $\gamma$-ray detectors, neutrino telescopes, and even radio telescopes can be used for indirect dark matter detection as well.

One of the most promising dark matter candidates are weakly interacting massive particles (WIMP) such as they are predicted within supersymmetric extensions of the Standard Model. Such particles have masses $m_{X} \gtrsim 100 \mathrm{GeV}$. If they are produced thermally, in order to reproduce the correct average dark matter density, their annihilation cross sections have to be $\langle\sigma v\rangle \sim 3 \times 10^{-26} \mathrm{~cm}^{3} / \mathrm{s}$ whereas large values are possible in case of non-thermal production.

Traditionally, indirect dark matter detection has focused on high energy emission,

specifically signatures in $\gamma$-rays $[4,5,6,7,8,9,10,11,12,13]$. High energy emission of neutrinos, the particle the most difficult to detect, can be used to establish conservative constraints on the total annihilation cross section, which are of the order $\sim 10^{-23} \mathrm{~cm}^{3} / \mathrm{s}[14]$. The fluxes of antiprotons and positrons from galactic dark matter annihilations has also been extensively used to constrain dark matter properties [15, 16, 17]. Furthermore, the synchrotron radiation emitted by dark matter annihilation products close to the Galactic centre has also been studied. It has been found that if the dark matter profile close to the central black hole is a spike formed by adiabatic accretion, typical dark matter annihilation cross sections within supersymmetric scenarios can lead to intensities comparable to the radio emission observed from the Galactic centre [18, 19]. It has further been shown that measurements of the radio flux away from the Galactic centre by the WMAP experiment strongly constrain the annihilation cross sections to values $\lesssim 10^{-25} \mathrm{~cm}^{3} / \mathrm{s}$ for $m_{X} \simeq 100 \mathrm{GeV}[20,21]$. A multi-wavelength analysis of dark matter annihilations from the Galactic centre has recently been performed in Ref. [22].

In the present paper we evaluate the diffuse synchrotron emission from the electrons and positrons produced by dark matter annihilation in the cosmological distribution of dark matter halos. We compute both its overall intensity and its angular power spectrum 
as well as the distribution of visible dark matter annihilation sources as a function of apparent luminosity. We will find that comparing the resulting signals with other backgrounds and foregrounds under conservative assumptions allows to test annihilation cross sections close to the natural scale $\langle\sigma v\rangle \sim 3 \times 10^{-26} \mathrm{~cm}^{3} / \mathrm{s}$.

In Sect. 2 we provide the general setup of our calculations. In Sect. 3 and 4, we apply it to astrophysical backgrounds and the dark matter induced signal, respectively. In Sect. 5 we compare the overall diffuse signal and its anisotropy to other foregrounds and discuss the resulting dark matter constraints, and in Sect. 6 we conclude. Finally, an appendix presents technical details of the calculations. We will use natural units in which $c=1$ throughout.

\section{Setup}

We consider a distribution of sources which emit a radio luminosity per frequency interval $L(\nu, \mathcal{P}, z)$ which depends on a parameter $\mathcal{P}$, on frequency $\nu$ and on redshift $z$. The energy flux per frequency interval and solid angle is then given by

$$
J(\nu)=\int d z \frac{d^{2} V}{d z d \Omega} \int d \mathcal{P} \frac{d n}{d \mathcal{P}}(\mathcal{P}, z) \frac{(1+z) L\left[\nu_{z}, \mathcal{P}, z\right]}{4 \pi d_{L}(z)^{2}},
$$

where for abbreviation we write $\nu_{z} \equiv(1+z) \nu,(d n / d \mathcal{P})(\mathcal{P}, z)$ is the co-moving volume density of objects per unit interval in the parameter $\mathcal{P}, d_{L}(z)$ is the luminosity distance, the factor $1+z$ comes from redshifting the frequency interval $d \nu$, and the co-moving volume per solid angle and redshift interval is

$$
\frac{d^{2} V}{d z d \Omega}=\frac{d_{L}(z)^{2}}{(1+z)^{2} H(z)}=\frac{r(z)^{2}}{H(z)} .
$$

Here, for a flat cosmological geometry, the Hubble rate is

$$
H(z)=H_{0}\left[\Omega_{n r}(1+z)^{3}+\Omega_{\Lambda}\right]^{1 / 2},
$$

$r(z)=\int_{t(z)}^{t(0)}(1+z) d t=\int d z^{\prime} / H\left(z^{\prime}\right)$ is the co-moving distance and $t(z)=\int_{0}^{z} d z^{\prime} /[(1+$ $\left.\left.z^{\prime}\right) H\left(z^{\prime}\right)\right]$ is cosmic time as function of redshift. Throughout this paper we will assume a flat, $\Lambda$ CDM Universe with the total non-relativistic matter density $\Omega_{n r}=0.279$ and the dark energy density $\Omega_{\Lambda}=1-\Omega_{n r} \simeq 0.701$ (all other contributions to the energy density are negligible) in units of the critical energy density $\rho_{c}=3 H_{0}^{2} /\left(8 \pi G_{\mathrm{N}}\right)$, where $G_{\mathrm{N}}$ is Newtons constant and $H_{0}=H(0)=100 h \mathrm{~km} \mathrm{~s}^{-1} \mathrm{Mpc}^{-1}$ with $h=0.701$ [1, 2].

In order to calculate the anisotropies we introduce an emissivity of squared power per frequency interval, $\mathcal{L}^{2}(\nu, k, z)$,

$$
\mathcal{L}^{2}(\nu, k, z)=\mathcal{L}_{1}^{2}(\nu, k, z)+\mathcal{L}_{2}^{2}(\nu, k, z),
$$

which, similar to the approach in Ref. [9], we split into the two parts $\mathcal{L}_{1}^{2}(\nu, k, z)$ and $\mathcal{L}_{2}^{2}(\nu, k, z)$ and which also depends on the co-moving wavenumber $k$. The first part is essentially Poisson noise and corresponds to the sum over squared luminosities,

$$
\mathcal{L}_{1}^{2}(\nu, k, z)=\int d \mathcal{P} \frac{d n}{d \mathcal{P}}(\mathcal{P}, z)\left[L\left(\nu_{z}, \mathcal{P}, z\right)|u(k, \mathcal{P})|\right]^{2},
$$


where in the following we define $\mathcal{F}_{f}(k) \equiv \int d^{3} \mathbf{r} e^{i \mathbf{k} \cdot \mathbf{r}} f(\mathbf{r})$ as the spatial Fourier transform of any function $f(\mathbf{r})$ and where $u(k, \mathcal{P})=\mathcal{F}_{u}(k, \mathcal{P})$ is the Fourier transform of the spatial emission density $u(\mathbf{r}, \mathcal{P})$ of an individual source, normalized to unity, $\int d^{3} \mathbf{r} u(\mathbf{r}, \mathcal{P})=1$. The second contribution to Eq. (4) is determined by the correlation between sources,

$$
\mathcal{L}_{2}^{2}(\nu, k, z)=P_{\operatorname{lin}}(k, z)\left[\int d \mathcal{P} \frac{d n}{d \mathcal{P}}(\mathcal{P}, z) L\left(\nu_{z}, \mathcal{P}, z\right) b(\mathcal{P}, z)|u(k, \mathcal{P})|\right]^{2},
$$

where $P_{\operatorname{lin}}(k, z)=\int d^{3} \mathbf{r} e^{i \mathbf{k} \cdot \mathbf{r}}(\delta \rho / \rho)(\mathbf{r}, z)$ is the linear power spectrum of the density fluctuations $(\delta \rho / \rho)(\mathbf{r}, z)$ and we have also introduced a bias factor $b(\mathcal{P}, z)$ of the sources with respect to the density field.

The angular power spectrum $C_{l}$ is given by

$$
C_{l}=\left\langle\left|a_{l m}\right|^{2}\right\rangle
$$

where

$$
a_{l m}=\int d \Omega[J(\nu, \Omega)-\langle J(\nu)\rangle] Y_{l m}^{*}(\Omega)
$$

in terms of the spherical harmonic functions $Y_{l m}(\Omega)$ and the intensity $J(\nu, \Omega)$ measured along direction $\Omega$. For a statistically isotropic sky this results in

$$
C_{l}=\int d z \frac{d^{2} V}{d z d \Omega} \frac{(1+z)^{2} \mathcal{L}^{2}\left(\nu_{z}, \frac{l}{r(z)}, z\right)}{\left[4 \pi d_{L}(z)^{2}\right]^{2}} .
$$

Using $d_{L}(z)=(1+z) r(z)$ and inserting Eq. (2) in Eqs. (1) and (9) finally gives

$$
J(\nu)=\frac{1}{4 \pi} \int \frac{d z}{(1+z) H(z)} \int d \mathcal{P} \frac{d n}{d \mathcal{P}}(\mathcal{P}, z) L\left(\nu_{z}, \mathcal{P}, z\right),
$$

and

$$
C_{l}=\frac{1}{(4 \pi)^{2}} \int d z \frac{\mathcal{L}^{2}\left(\nu_{z}, \frac{l}{r(z)}, z\right)}{d_{L}(z)^{2} H(z)} .
$$

Formally, for point-like sources, the integral over redshift in Eq. (11) is divergent at $z \rightarrow 0$. In practice this is regularized by the fact that the nearest source has some minimal distance and that one can subtract the most luminous point sources which are also the nearest sources. In addition, the integral is regularized by the spatial extent of the sources, represented by the factor $|u(k, \mathcal{P})|^{2}$ in Eqs. (5) and (6). The role of these effects in practical calculations will be discussed in Sect. 5.2.

\section{Astrophysical Sources}

For astrophysical sources, $\mathcal{P}$ can be identified with the radio luminosity $L_{\nu_{0}}$ at some fixed frequency $\nu_{0}$. Eq. (10) then simplifies to

$$
J(\nu)=\frac{1}{4 \pi} \int \frac{d z}{(1+z) H(z)} \int^{L_{\mathrm{cut}}(z)} d L_{\nu_{0}} L_{\nu_{0}} \frac{d n}{d L_{\nu_{0}}}\left(L_{\nu_{0}}, z\right) \frac{L\left(\nu_{z}\right)}{L_{\nu_{0}}},
$$

where $L_{\text {cut }}(z)=4 \pi d_{L}(z)^{2} S_{\text {cut }} /(1+z)$ is the intrinsic luminosity corresponding to the apparent point source flux $S_{\text {cut }}$ above which we consider the source to be resolvable and 
thus subtractable from the diffuse background. For the multipoles Eqs. (5) and (6) can then be written as

$\mathcal{L}_{1}^{2}(\nu, k, z)=\int^{L_{\mathrm{cut}}(z)} d L_{\nu_{0}} L_{\nu_{0}}^{2} \frac{d n}{d L_{\nu_{0}}}\left(L_{\nu_{0}}, z\right)\left[\frac{L\left(\nu_{z}\right)}{L_{\nu_{0}}} u(k, z)\right]^{2}$

and

$\mathcal{L}_{2}^{2}(\nu, k, z)=P_{\text {lin }}(k, z)\left[\int^{L_{\mathrm{cut}}(z)} d L_{\nu_{0}} L_{\nu_{0}} \frac{d n}{d L_{\nu_{0}}}\left(L_{\nu_{0}}, z\right) \frac{L\left(\nu_{z}\right)}{L_{\nu_{0}}} u(k, z) b\left(L_{\nu_{0}}, z\right)\right]^{2}$,

respectively. For the luminosity functions $d n / d L_{\nu_{0}}$ of normal and radio galaxies we will use the expressions given in Ref. [23].

\section{Dark Matter Annihilation}

For annihilation of dark matter with mass $m_{X}$ and phase space averaged annihilation cross section times velocity $\langle\sigma v\rangle, \mathcal{P}$ can be identified with the mass $M$ of dark matter halos. We then follow the approach of Ref. [18] and write

$$
L(\nu, M)=\frac{\langle\sigma v\rangle}{2 m_{X}^{2}} \mathcal{E}(\nu, M),
$$

where we define $\mathcal{E}(\nu, M)$ as a quantity which does not depend on annihilation cross section or mass of the dark matter particles,

$\mathcal{E}(\nu, M)=\frac{\sqrt{3} e^{3}}{m_{e}} \int d^{3} \mathbf{r} \rho_{h}^{2}(\mathbf{r}) B(\mathbf{r}) \int_{m_{e}}^{m_{X}} d E \frac{Y_{e}(>E)}{P_{\mathrm{syn}}(E)+P_{\mathrm{IC}}(E)} F\left[\frac{\nu}{\nu_{c}(E)}\right]$.

In Eq. (16), $e$ and $m_{e}$ are the electron charge and mass, respectively, $\rho_{h}(\mathbf{r})$ is the dark matter halo density profile, $B(\mathbf{r})$ is the local magnetic field strength,,$Y_{e}(>E)$ is the multiplicity per annihilation of electrons and positrons with energies larger than $E, P_{\text {syn }}(E)=2 e^{4} B^{2} E^{2} /\left(3 m_{e}^{4}\right)=\left(16 e^{4} \pi / 3\right) u_{B} E^{2} / m_{e}^{4}$ is the total synchrotron emission power of one electron of energy $E$ in a magnetic field of strength $B$, corresponding to an energy density $u_{B}=B^{2} /(8 \pi)$, and $P_{\mathrm{IC}}(E)=\left(16 e^{4} \pi / 3\right) u_{\gamma} E^{2} / m_{e}^{4}$ is the energy loss rate at energy $E$ due to inverse Compton scattering on a low energy photon field of energy density $u_{\gamma}$. Furthermore, we use the function

$$
F(x)=x \int_{x}^{\infty} K_{5 / 3}(y) d y,
$$

in Eq. (16), with the critical frequeny

$$
\nu_{c}(E)=\frac{3}{4 \pi} \frac{e B}{m_{e}}\left(\frac{E}{m_{e}}\right)^{2} .
$$

In the following we use the approximation [24]

$$
F(x) \simeq \delta[x-0.29]
$$

such that Eq. (16) can be simplified to

$$
\mathcal{E}(\nu, M) \simeq \frac{9}{8}\left(\frac{m_{e}^{3}}{0.29 \pi}\right)^{1 / 2} \frac{Y_{e}\left[>E_{c}(\nu)\right]}{\nu^{1 / 2}} I(M)
$$


where

$$
I(M)=\int d^{3} \mathbf{r} \frac{\rho_{m}^{2}(\mathbf{r})}{(e B)^{1 / 2}(\mathbf{r})} \frac{1}{1+u_{\gamma}(\mathbf{r}) / u_{B}(\mathbf{r})},
$$

and the critical energy $E_{c}(\nu)$ is the inversion of Eq. (18),

$$
E_{c}(\nu)=\left(\frac{4 \pi}{3 \cdot 0.29} \frac{m_{e}^{3}}{e} \frac{\nu}{B}\right)^{1 / 2}=5.9\left(\frac{\nu}{1 \mathrm{GHz}}\right)^{1 / 2}\left(\frac{B}{6 \mu \mathrm{G}}\right)^{-1 / 2} \mathrm{GeV} .
$$

In Eq. (20) we neglect the magnetic field dependence of $Y_{e}\left[>E_{c}(\nu)\right]$. For $m_{X} \gtrsim 100 \mathrm{GeV}$, $B \gtrsim$ a few micro-Gauss and $\nu \sim 1 \mathrm{GHz}$, the parameters we are interested in, this is a good approximation because the critical energy $E_{c}\left(\nu_{z}\right) \lesssim m_{X} / 10$. Typical values for these parameters are $Y_{e} \simeq 10$ [18]. This corresponds to a fraction $f_{e} \simeq 0.3$ of the total annihilation energy going into pairs. The energy fraction going into pairs of energy above $E$ can be expressed in terms of $Y_{e}(>E)$ as

$$
f_{e}(E)=\frac{-1}{2 m_{X}} \int_{E}^{m_{X}} d E^{\prime} E^{\prime} \frac{d Y_{e}}{d E}\left(E^{\prime}\right) \leq 1
$$

With the above expressions we can rewrite Eq. (10) as

$J(\nu)=\frac{\langle\sigma v\rangle}{2 m_{X}^{2}} \frac{9}{32 \pi}\left(\frac{m_{e}^{3}}{0.29 \pi \nu}\right)^{1 / 2} \int \frac{d z}{(1+z)^{3 / 2} H(z)} \int d M \frac{d n}{d M}(M, z) Y_{e}\left[>E_{c}\left(\nu_{z}\right)\right] I(M)$.

Furthermore, we can redefine $\mathcal{L}_{1}^{2}$ and $\mathcal{L}_{2}^{2}$ from Eqs. (5) and (6) by extracting constant factors and write

$\mathcal{L}_{1}^{2}(\nu, k, z)=\int d M \frac{d n}{d M}(M, z)\left(Y_{e}\left[>E_{c}\left(\nu_{z}\right)\right] I(M)|u(k, M)|\right)^{2}$

and

$\mathcal{L}_{2}^{2}(\nu, k, z)=P_{\text {lin }}(k, z)\left(\int d M \frac{d n}{d M}(M, z) Y_{e}\left[>E_{c}\left(\nu_{z}\right)\right] I(M) b(M, z)|u(k, M)|\right)^{2}$,

where $u(k, M)$ relates to the halo profile, $u(k, M)=\int d^{3} \mathbf{r} e^{i \mathbf{k} \cdot \mathbf{r}} \rho_{h}^{2}(\mathbf{r})(e B)^{-1 / 2}(\mathbf{r}) / I(M)$. With these quantities we can now write

$$
C_{l}=\frac{81 m_{e}^{3}}{1024 \cdot 0.29 \pi^{3} \nu}\left(\frac{\sigma v}{m_{X}^{2}}\right)^{2} \int d z \frac{\mathcal{L}_{1}^{2}\left(\nu, \frac{l}{r(z)}, z\right)+\mathcal{L}_{2}^{2}\left(\nu, \frac{l}{r(z)}, z\right)}{(1+z) d_{L}(z)^{2} H(z)} .
$$

Details about the quantities that enter these expressions are given in Appendix A.

Eq. (27) can also be obtained as follows: Limber's equation relates the twodimensional angular power spectrum $P_{2}(l)$ to the three-dimensional power spectrum $P_{3}(k)$ in the flat sky approximation [25]: Given a three-dimensional statistically random field $f(\mathbf{r})=f(\Omega, r)$, one considers the observation at $\mathbf{r}=0$ of the projection

$$
P(\Omega)=\int d r w(r) f(\Omega, r)
$$

with some given radial weight function $w(r)$, where $r$ is the co-moving distance. If the field $f$ fluctuates on scales much smaller than the characteristic scale over which $w(r)$ varies, then we have

$$
C_{l} \simeq \int d r \frac{w^{2}(r)}{r^{2}} P_{f}(l / r, z(r))
$$


where $P_{f}(l / r, z)$ is the power spectrum of $\left\langle f\left(\Omega_{1}, r\right) f\left(\Omega_{2}, r\right)\right\rangle$ at the co-moving wavenumber $k=l / r$.

Neglecting the variation of the magnetic field $B$ within the halo regions contributing most to the annihilations, the radio intensity Eq. (24) along a given direction $\Omega$ can be written as

$J(\nu, \Omega)=\frac{\langle\sigma v\rangle}{m_{X}^{2}} \frac{9 \rho_{m}}{64 \pi \nu^{1 / 2}}\left(\frac{m_{e}^{3}}{0.29 \pi e B}\right)^{1 / 2} \int d z \frac{(1+z)^{3 / 2}}{H(z)} Y_{e}\left[>E_{c}\left(\nu_{z}\right)\right] \frac{[1+\delta(z, \Omega)]^{2}}{1+u_{\gamma} / u_{B}}$,

where $\rho_{m}=\Omega_{m} \rho_{c}$ is the average dark matter density at zero redshift, and $\delta=\delta \rho / \rho$ is the relative overdensity. Because the dominant contribution comes from the dark matter halos, where $\delta \gg 1$, we can approximate $(1+\delta)^{2} \simeq \delta^{2}$. Assuming a constant $B$ and a constant optical photon field of density $u_{\mathrm{op}} \simeq 5 \mathrm{eV} \mathrm{cm}^{-3}$, we can write the factor $\left(1+u_{\gamma} / u_{B}\right)^{-1}=\left[1+u_{\mathrm{op}} / u_{B}+u_{0}(1+z)^{4} / u_{B}\right]^{-1}$, where $u_{0}$ is the CMB energy density at $z=0$. This factor effectively cuts off the redshift integration at $z \simeq 2$. Since $E_{c}\left(\nu_{z}\right)$ varies little over this redshift range, we can then further simplify Eq. (30) to

$J(\nu, \Omega) \simeq \frac{Y_{e}\left[>E_{c}(\nu)\right]\langle\sigma v\rangle}{m_{X}^{2}} \frac{9 \rho_{m}^{2}}{64 \pi \sqrt{e B \nu}}\left(\frac{m_{e}^{3}}{0.29 \pi}\right)^{1 / 2} \int d z \frac{(1+z)^{3 / 2} \delta^{2}(z, \Omega)}{H(z)\left[1+\frac{u_{\mathrm{op}}}{u_{B}}+\frac{u_{0}}{u_{B}}(1+z)^{4}\right]}$.

Comparing this with Eq. (28), we can use

$$
f=\delta^{2}-\left\langle\delta^{2}\right\rangle
$$

for the random field and the weight function is

$$
w(z)=\frac{Y_{e}\left[>E_{c}(\nu)\right]\langle\sigma v\rangle}{m_{X}^{2}} \frac{9 \rho_{m}^{2}}{64 \pi \sqrt{e B \nu}}\left(\frac{m_{e}^{3}}{0.29 \pi}\right)^{1 / 2} \frac{(1+z)^{3 / 2}}{1+\frac{u_{\mathrm{op}}}{u_{B}}+\frac{u_{0}}{u_{B}}(1+z)^{4}} .(33
$$

The power spectrum $P_{f}(k, z)$ appearing in Eq. (29) is then the Fourier transform of the two-point correlation function of $f$ in real space. Following Ref. [8], $P_{\delta^{2}}(k, z)$ can be written as the sum of a one-halo and a two-halo term, $P_{\delta^{2}}(k, z)=P_{\delta^{2}}^{1 h}(k, z)+P_{\delta^{2}}^{2 h}(k, z)$, with

$$
\begin{aligned}
P_{\delta^{2}}^{1 h}(k, z) & =\int_{M_{\text {min }}}^{M_{\mathrm{cut}}(z)} d M \frac{d n}{d M}\left[\mathcal{F}_{\delta^{2}}(k, M, z)\right]^{2}=\int_{M_{\text {min }}}^{M_{\mathrm{cut}}(z)} d M \frac{d n}{d M}\left(\frac{A_{b} \mathcal{F}_{\rho_{h}^{2}}(k, M, z)}{\rho_{m}^{2}(1+z)^{6}}\right)^{2} \\
P_{\delta^{2}}^{2 h}(k, z) & =P_{\operatorname{lin}}(k)\left[\int_{M_{\min }}^{M_{\mathrm{cut}}(z)} d M \frac{d n}{d M} b(M) \mathcal{F}_{\delta^{2}}(k, M, z)\right]^{2} \\
& =P_{\text {lin }}(k)\left[\int_{M_{\text {min }}}^{M_{\mathrm{cut}}(z)} d M \frac{d n}{d M} b(M)\left(\frac{A_{b} \mathcal{F}_{\rho_{h}^{2}}(k, M, z)}{\rho_{m}^{2}(1+z)^{6}}\right)\right]^{2},
\end{aligned}
$$

where $M_{\min }$ is the minimal halo mass and $M_{\text {cut }}(z)$ is the halo mass corresponding to the apparent point source flux $S_{\text {cut }}$ above which we consider the source to be resolvable and thus subtractable from the diffuse background. Furthermore, $A_{b}$ is a boost factor which accounts for possible substructure in the halos. The average of the clumping factor appearing in Eq. (31) is given by

$$
\left\langle\delta^{2}(z)\right\rangle=\frac{A_{b}}{\rho_{m}^{2}(1+z)^{6}} \int_{M_{\min }}^{M_{\mathrm{cut}}(z)} d M \frac{d n}{d M} \times \int d V_{h} \rho_{h}^{2}(\mathrm{r}, M, z),
$$


where $d V_{h}$ is the halo volume element.

A generic form for the halo mass function $d n / d M$ appearing in the equations above was first proposed by Press \& Schechter (PS) [26]; a modified version of this form is given by Sheth and Tormen [27] (ST). When comparing the results obtained from these two forms, we find differences by factors less than 2. Thus, we adopt the PS formula throughout our paper.

Current knowledge of the dark matter density distribution mostly comes from Nbody simulations, and the universal dark matter profile firstly proposed is the NavarroFrenk-White (NFW) model [28]. Combining Eq. (A.14) with Eq. (A.17) in Appendix A, in this model the dark matter profile within each halo can be written as

$$
\rho_{h}(r)=\frac{\Delta_{c}(z)}{3} \frac{c^{3}}{\ln (1+c)-c /(1+c)} \frac{\rho_{m}(z)}{r / r_{s}\left(1+r / r_{s}\right)^{2}},
$$

where $r_{s}$ is a characteristic radius, and the concentration parameter $c$ is defined as the ratio of $r_{s}$ and the virial radius $r_{v}, c \equiv r_{v} / r_{s}$, see also Appendix A for more details. Note that $r_{s}$ is not a free parameter, but depends on $M$ and $c$ because $r_{v}$ is related to $M$ via $M=4 \pi \Delta_{c}(z) \rho_{m}(z) / 3$, where $\Delta_{c}(z)$ in an Einstein-de Sitter Universe is about $18 \pi^{2}$. With the above definition, $c$ and $M$ completely determine the dark matter distribution of a given halo. The minimal halo mass is still rather uncertain. The value $M_{\min }=10^{-6} M_{\odot}[29]$ is close to the free-streaming mass $[30,31,32]$, below which there are no fluctuations in the dark matter density to form a halo. Note that the magnetic field may be much smaller than micro Gauss scales in such small halos. In contrast, the value $M_{\text {min }}=10^{6} M_{\odot}$ roughly corresponds to the minimal mass of dwarf galaxies which are known to contain micro Gauss scale magnetic fields [33]. We, therefore, choose $M_{\text {min }}=10^{6} M_{\odot}$ as fiducial value in the following, noting that the dark matter signal would increase by only a factor about two for $M_{\min }=10^{-6} M_{\odot}$. We will furthermore use $B=10 \mu \mathrm{G}$ as fiducial value for the magnetic field. This is a realistic value given that most annihilations occur in the densest regions where also magnetic fields are somewhat larger than typical average galactic fields.

The clumping factor is very sensitive to the concentration parameter, namely $\propto c^{3}$. N-body simulations indicate that the concentration has a log-normal distribution [34] with a median value of

$$
c(M, z)=4 \frac{1+z_{c}}{1+z},
$$

where the collapse redshift $z_{c}$ is implicitly given by the relation $M_{*}\left(z_{c}\right)=0.01 M$, where $M_{*}(z)$ is the mass scale at which $\sigma\left(M_{*}, z\right)=\delta_{c}$. How the concentration parameter depends on halo mass and redshift is still an open question. One can extrapolate Eq. (38) to minimal halo masses $M \sim 10^{-6} M_{\odot}$. When comparing the parameterization Eq. (38) with high resolution simulations [29] we find that it gives realistic values for the minimum halo mass. It is a conservative estimate because at $z \simeq 0$ it gives values $c \sim 70$ for the minimum halo mass which is significantly smaller than other parameterizations $[35,36]$.

Recent studies show that dark matter halos exhibit considerable substructure [37, $38,39,40,41,42,43,44,45,46,47]$. The total mass of these substructures only account 
for about $10 \%$ of the host halo, but they can give an extra boost factor $A_{b} \sim 10$ for dark matter annihilation. Some studies show that if one takes into account substructure and assumes a cuspy center slope [48, 49, 50], the theoretical prediction can well explain the excess of high energy positrons and the diffuse $\gamma$ - ray background observed by the Heat [51, 52] and EGRET [53, 54] experiments, respectively. The subhalos follow a certain mass and redshift distribution which is still unknown. Therefore, to be conservative we assume the NFW halo model and simply parametrize any possible boost factor with the parameter $A_{b} \sim 10$. The substructures occur on small scales and do not influence the power spectrum in the range we are interested, $l \lesssim 10^{4}$.

\section{Results}

\subsection{Diffuse Radio Emission}

The cosmic microwave background (CMB) dominates the radio sky at frequencies above $\simeq 1 \mathrm{GHz}$, whereas astrophysical sources such as normal galaxies and radio galaxies dominate at lower frequencies down to $\mathrm{kHz}$ frequencies [23]. Recently it was argued that synchrotron emission of strong intergalactic shocks can also significantly contribute to the diffuse extragalactic radio below $500 \mathrm{MHz}[55,56]$.

Using the formuli developed in Sect. 4, we now evaluate the contribution of synchrotron emission from pairs produced by dark matter annihilation in the magnetic fields of dark matter halos. We consider neutralinos as dark matter candidate, and for the following figures we assume a neutralino mass of $100 \mathrm{GeV}$ and a total annihilation

cross section of $\langle\sigma v\rangle=3 \times 10^{-26} \mathrm{~cm}^{3} / \mathrm{s}$, fixed for reproducing the correct relic density for thermal relics. We also assume that the average total number of electrons and positrons per annihilation is $Y_{e} \simeq 10$, and that the halo substructure implies a boost factor $A_{b} \simeq 10$. We compare the resulting dark matter signal with astrophysical contributions to the diffuse background that can be computed from the expressions in Sect. 3.

For astrophysical sources the diffuse radio background is likely dominated by normal galaxies and radio galaxies. To estimate the contributions from these sources, we follow Ref. [23], which use the observed correlation between the radio and infra-red flux of galaxies. This approach assumes that the radio emission is related to the star formation and is sensitive to the redshift evolution of the sources, but can explain the observed radio background quite well.

Following the above assumptions, in Fig. 1, we show the different contributions to the average diffuse radio intensity. For astrophysics sources, normal galaxies contribute more than radio galaxies. This is because although the individual radio galaxy is brighter than a normal galaxy on average, this is overcompensated by the larger number of normal galaxies. Also shown in Fig. 1 is a possible contribution from intergalactic shocks [55, 56] normalized such that its angular power spectrum is comparable to the one of the Galactic foreground, see Sect.5.2.

Of course, the CMB absolutely dominates the radio sky in the wide range from $\nu \simeq$ 


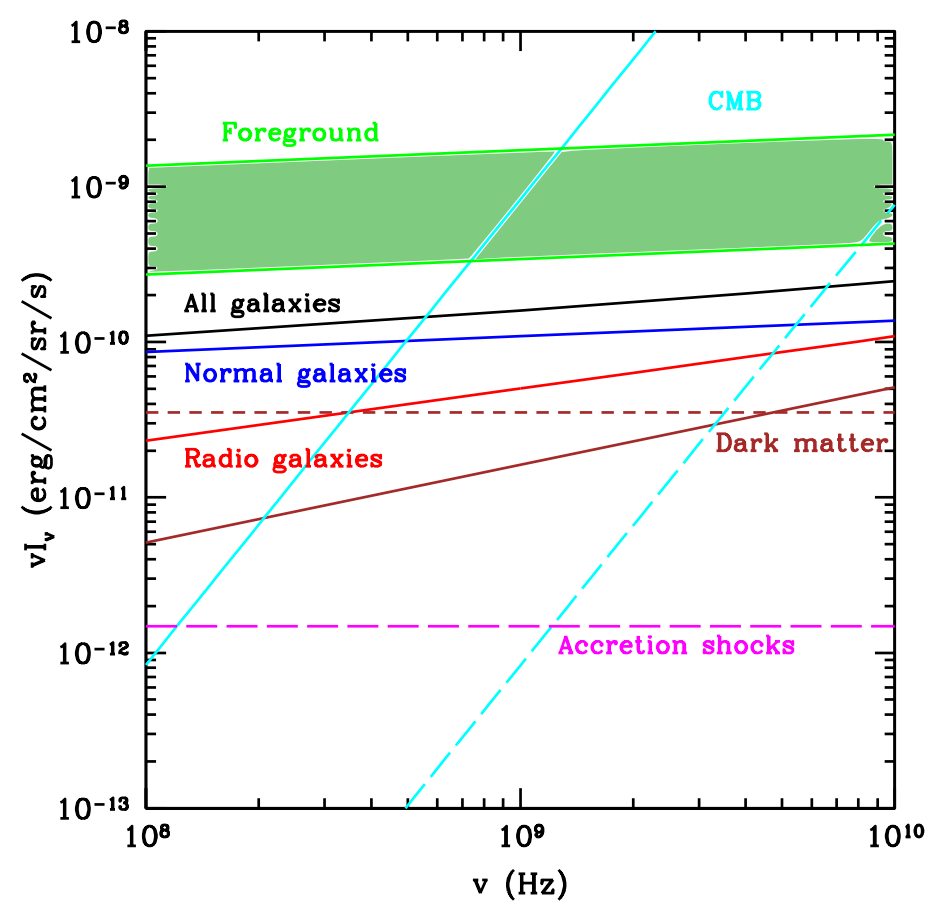

Figure 1. The average diffuse background flux intensity with no point-source removal. Contributions from normal galaxies (blue curve), radio galaxies (red curve), from radio and normal galaxies combined (black curve), and from a scenario for radio emission from galaxy cluster shocks (magenta curve) [56] (see text for the normalization) are compared to our fiducial dark matter annihilation scenario with $m_{X}=100 \mathrm{GeV}$, $\langle\sigma v\rangle \sim 3 \times 10^{-26} \mathrm{~cm}^{3} / \mathrm{s}, A_{b}=10, B=10 \mu \mathrm{G}, M_{\min }=10^{6} M_{\odot}$ (brown curves). Here, the solid brown curve is for $Y_{e}=10$, while the dashed brown curve is for $Y_{e}(E) \simeq m_{X} / E$. Also shown is the CMB background (cyan solid curve) as well as its subtractable part, determined by uncertainties of the absolute CMB temperature (dotted cyan curve). The Galactic foreground at Galactic latitude $b>20^{\circ}$ is shown as the green band within uncertainties.

$1 \mathrm{GHz}$ to a few hundred GHz [57], and above these frequencies Galactic foregrounds such as dust emission dominates. Since the CMB is a black body radiator its contribution to the solid angle averaged radio flux can be subtracted up to the uncertainty of its average absolute temperature. Currently the CMB temperature is measured to $2.725 \pm 0.001$ $\mathrm{K}$ [58]. We convert this temperature uncertainty into an intensity of CMB confusion noise. Fig. 1 shows that this confusion noise dominates other astrophysical backgrounds and the diffuse signal of our fiducial dark matter scenario at $\nu \gtrsim 4 \mathrm{GHz}$. At lower frequencies the dark matter signal $\nu J(\nu)$ tends to decrease as $\sqrt{\nu}$ for $Y_{e} \simeq$ const., see Eq. (31), whereas the background from normal galaxies tends to be flat, see Fig. 1. There is thus an optimal window at frequencies $\nu \sim 1 \mathrm{GHz}$ where dark matter annihilation signatures can be detected and where self-absorption is negligible. Constraints on dark matter parameters can, therefore, only be established for annihilation cross sections about a factor ten higher than the fiducial cross section required for thermal dark matter.

In addition, there are three diffuse foregrounds from our Galaxy in the frequency 
range we are interested: The first is synchrotron radiation emitted by high energy electrons gyrating in the Galactic magnetic field, the second is free-free emission from the thermal bremsstrahlung from hot $\left(\geq 10^{4} \mathrm{~K}\right)$ electrons produced in the interstellar gas by the Galactic UV radiation field, and the third foreground is dust emission which arises from the thermal re-radiation of absorbed stellar light. Fig. 1 shows that these foregrounds tend to dominate the astrophysical backgrounds and the dark matter signal in the fiducial scenario.

Can we test the properties of dark matter more powerfully? The absolute CMB temperature is difficult to measure more precisely than to the current permille level, because of inevitable systematic errors. Small-scale temperature fluctuations $\Delta T / T \sim$ $10^{-5}$ have been seen by the COBE and WMAP satellites because temperature differences can be measured more precisely since systematic errors cancel in measurements of temperature differences. Furthermore, if the Galactic foregrounds have a smooth directional dependence, they may pose less of a contamination when considering the anisotropy of the radio sky. We, therefore, consider in the following the angular power spectra of the radio sky in order to see if it can provide further tests of dark matter properties.

\subsection{Anisotropy}

Whereas the diffuse average radio flux provides only one number at a given frequency to compare with other astrophysical and cosmological backgrounds, potentially much more information is contained in the angular power spectrum. For example, the power spectrum as a function of angular scale tends to be different for dark matter annihilation and astrophysical sources because the contribution of the latter to the diffuse radio flux is dominated by fewer bright sources. Our goal in this section is whether this can provide dark matter signatures or constraints on mass and annihilation cross section.

Before calculating the angular power spectra, we discuss their qualitative behaviors. The angular power spectrum $C_{l}=C_{l}^{1 h}+C_{l}^{2 h}$ can be divided into one-halo $\left(C_{l}^{1 h}\right)$ and two-halo $\left(C_{l}^{2 h}\right)$ terms, corresponding to the two contributions Eqs. (5) and (6) to Eq. (4), and thus to Eq. (11). The two-halo term arises from the correlation between distinct halos which is described by the linear power spectrum. The one-halo term represents correlation within the same halo. Both one-halo and two-halo term are proportional to $|u(k, \mathcal{P})|^{2}$, the square of the Fourier transform of the spatial emission profile. At large angular scales, $|u(k, \mathcal{P})| \sim 1$, such that $C_{l}^{1 h}$ is essentially independent of $l$. The one-halo term is thus sometimes called Poisson noise. At scales comparable to the size of the source, $|u(k, \mathcal{P})|^{2}$ starts to become suppressed. Therefore, both one-halo and two-halo terms are expected to be suppressed for multipoles $l$ larger than the typical distance to the source divided by the linear source size. The two-halo term is furthermore proportional to the linear power spectrum which is also suppressed for

co-moving wavenumbers $k \gtrsim 0.03 \mathrm{Mpc}^{-1}$. Therefore, the ratio of the two-halo term to the one-halo term is suppressed for $l \gtrsim 0.03 \mathrm{Mpc}^{-1} r_{\mathrm{H}} \simeq 100$, where $r_{\mathrm{H}} \simeq 3000 \mathrm{Mpc}$ is 
the Hubble scale. The one-halo term eventually dominates at very small angular scales.

In Eq. (11), for point-like sources, formally the one-halo term $C_{l}^{1 h}$ would diverge for $z_{\text {min }} \rightarrow 0$, whereas the two-halo term Eq. (6) is regularized by the linear power spectrum $P_{\operatorname{lin}}(k, z)$, which is suppressed at large $k=l / r(z)$. This is because the flux of nearby sources of a given luminosity diverges. We can ignore such sources because they can be identified as individual bright sources and be removed from the background flux in actual observations. We can remove sources with intrinsic luminosity $L_{\text {cut }}(z) \geq 4 \pi d_{L}(z)^{2} S_{\text {cut }} /(1+z)$, corresponding to the point-source sensitivity $S_{\text {cut }}$ of the telescope. Alternatively, one can regularize Eq. (11) by integrating from some finite minimum distance corresponding to the typical distance to the nearest source, $r_{\min } \sim 1 \mathrm{Mpc}$. Furthermore, Eq. (11) is also formally regularized at $z_{\min } \rightarrow 0$ by the spatial extent of nearby sources, described by $|u(k, \mathcal{P})|^{2}$. For the NFW profile, the mass of the halo within distance $r$ from the halo centre increases as $r^{2}$ up to $r=r_{s}$, and then increases logarithmically between $r_{s}$ and $r_{v}$ since $\rho_{h}(r) \propto r^{-3}$, see Eq. (37). Therefore, the dominant contribution to the halo mass comes from $r<r_{s}$. Similarly, for $r<r_{s}$ the annihilation signal increases as $r$, but between $r_{s}$ and $r_{v}$ increases only as $r_{s}^{-3}-r^{-3}$. Assuming the emission traces $\rho_{h}$ for astrophysical emission processes and $\rho_{h}^{2}$ for dark matter annihilation, the Fourier transforms of these dependencies then give $u(k, \mathcal{P}) \propto k^{-\gamma}$ for $k \gg r_{s}^{-1}$, with $\gamma=2$ for astrophysical emission and $\gamma=1$ for dark matter, see Appendix A.6 for more details. Since $k=l / r(z)$, and thus $|u[l / r(z), \mathcal{P}]|^{2} \propto r(z)^{2 \gamma}$, the one-halo term in Eq. (11) diverges only for $\gamma \leq 0.5$. Therefore, under our assumptions for the emission profile, Eq. (11) is convergent even without cut-offs in either $r_{\min }$ or the apparent luminosity. Since nevertheless in particular the one-halo term is quite sensitive to nearby sources, in the following we study its dependence on $S_{\text {cut }}$ and $r_{\text {min }}$.

Fig. 2 shows the dependence of the two-halo term on $S_{\text {cut }}$. According to Eq. (6), the two-halo term scales with the square of the average flux. Since the apparent luminosity of radio galaxies can be of the order of a Jansky ( $1 \mathrm{Jy}=10^{-23} \mathrm{erg} \mathrm{cm}^{-2} \mathrm{~Hz}^{-1} \mathrm{~s}^{-1}$ ), the twohalo term from radio galaxies starts to decline when we cut sources being less luminous than a critical luminosity below a Jansky. In contrast, the contribution of normal galaxies which are much less luminous than radio galaxies starts to decline only when we cut sources more luminous than $\simeq 10 \mu \mathrm{Jy}$. The contributions of dark matter halos to the dark matter annihilation signal is basically unaffected by any source removal, even to luminosities down to $\sim 1 \mu \mathrm{Jy}$. This is easy to explain: In our fiducial scenario the largest dark halos of about $10^{14} M_{\odot}$ produce only about $1.3 \times 10^{38} \mathrm{erg} / \mathrm{s}$ at $2 \mathrm{GHz}$ from dark matter annihilation, far less than the typical radio luminosity of galaxies of about $2 \times 10^{40} \mathrm{erg} / \mathrm{s}$. As a result, removing bright sources increases the contribution of dark matter annihilation to the two-halo term relative to the contribution from astrophysical sources.

Next we discuss the one-halo term. The one-halo term is more sensitive to the cut-offs in apparent luminosity $S_{\text {cut }}$ and to the minimal distance $r_{\text {min }}$ than the two-halo term because of two reasons: First, the two-halo term Eq. (6) is the square of an integral 


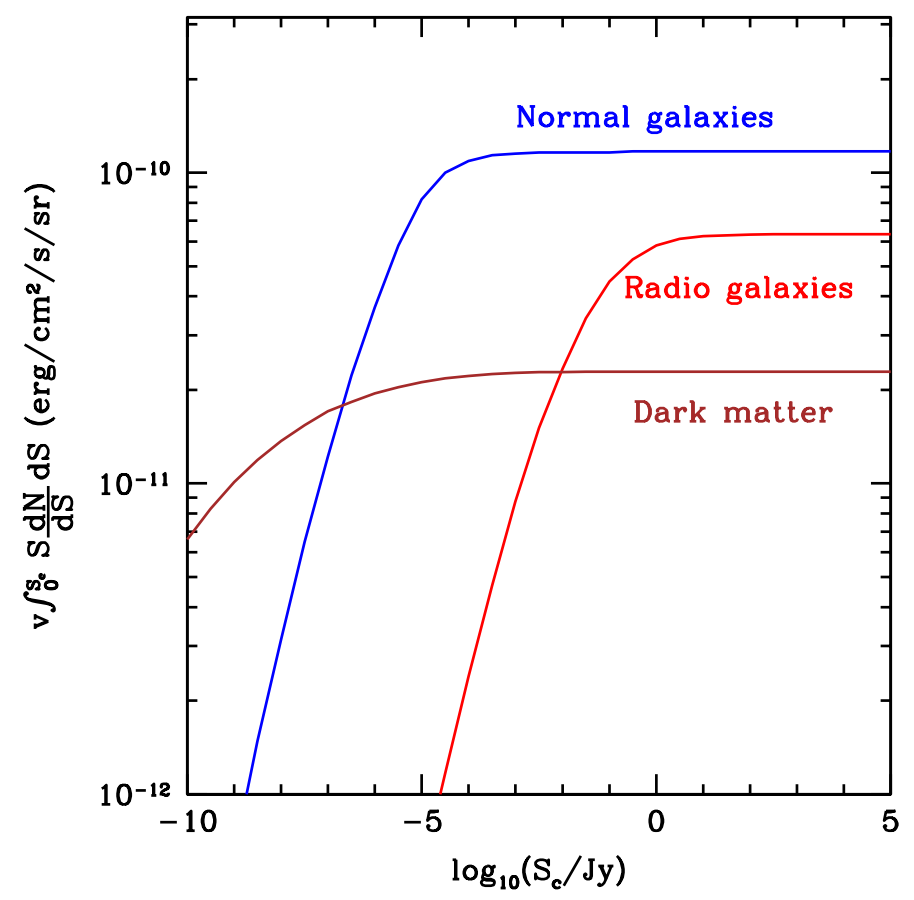

Figure 2. The cumulative contribution of sources of apparent luminosity $S$ smaller than $S_{\text {cut }}$ to the two-halo term at $2 \mathrm{GHz}$. The red, blue and brown lines represent the contribution from radio galaxies, normal galaxies, and dark matter (fiducial scenario with $\left.Y_{e}=10\right)$, respectively.

of luminosities, whereas the one-halo term Eq. (5) is essentially Poisson noise and thus proportional to an integral of squared luminosities, which makes the contribution from bright sources more important. Second, the two-halo term is further regularized by the linear power spectrum at large $k=l / r(z)$. In Fig. 3 we show the cumulative contribution of sources dimmer than $S_{\text {cut }}$ to $C_{l}^{1 h}$. Similarly to the two-halo term shown in Fig. 2 , the contribution of radio galaxies and ordinary galaxies decreases rapidly below $\simeq 1 \mathrm{Jy}$ and $10 \mu \mathrm{Jy}$, respectively, whereas the contribution of dark matter annihilation is affected less by source removal. Nevertheless, the contribution of bright sources is now much larger than for the two-halo term, as expected, and the one-halo term continues to rise with inclusion of brighter sources. On the other hand, practically one should cut off the integral at some minimal distance $r_{\min } \simeq 1 \mathrm{Mpc}$ within which there are essentially no bright sources. Since sources at small distance appear bright, the cut-off in luminosity and minimal distance is of course to some extent degenerate, as confirmed by Fig. 3. For radio galaxies, removal above $\simeq 10^{5} \mathrm{Jy}$ is equivalent to restricting to distances larger than 1 Mpc. For ordinary galaxies, cutting at a minimal distance $r_{\min }=1 \mathrm{Mpc}$ is equivalent to removing sources brighter than $0.1 \mathrm{Jy}$. Since observational sensitivities are considerably better than these luminosities, cutting at $r_{\min } \simeq 1 \mathrm{Mpc}$ does, therefore, not introduce any significant uncertainties. Note that in Fig. 2 dark matter dominates the two-halo terms if all sources above $\simeq 0.1 \mu \mathrm{Jy}$ are removed, while in Fig. 3 it would 


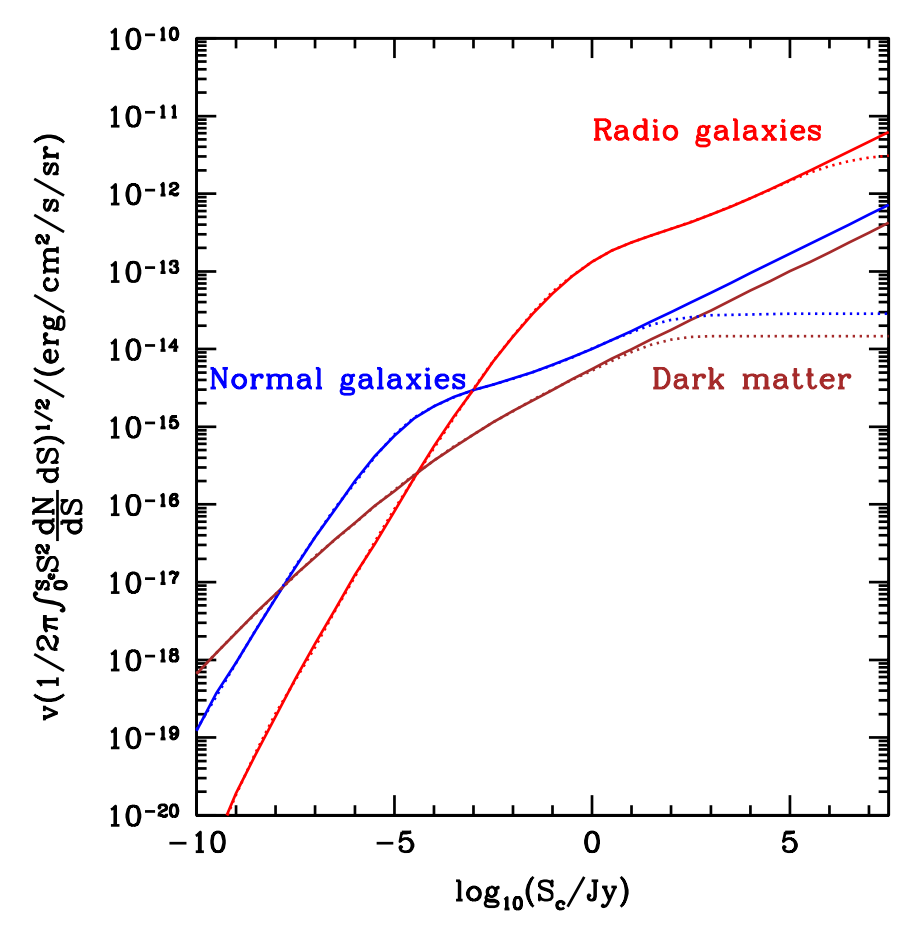

Figure 3. The cumulative contribution of sources of apparent luminosity $S$ smaller than $S_{\text {cut }}$ to the one-halo (Poisson) term at $2 \mathrm{GHz}$. The solid and dotted curve represent the cases of $r_{\min }=0$ and $r_{\min }=1 \mathrm{Mpc}$, respectively. Color keys are as in Fig. 2.

dominate the one-halo terms only for unrealistically small cut-off luminosities $\lesssim 1$ nJy. This is because the one-halo term is much more sensitive to bright sources than the two-halo term and because the dark matter contribution consists of dimmer sources than ordinary astrophysical sources.

The angular power spectra of the radio background at $2 \mathrm{GHz}$ produced by galaxies and by our fiducial dark matter scenario are shown in Figs. 4, 5, and 6 for different source removal cuts. Based on the above discussion, the qualitative behavior of the one- and two-halo terms can be easily understood: In Fig. 4 we assume galaxies to appear pointlike and we remove sources brighter than $0.1 \mathrm{mJy}$. The one-halo terms from these sources thus increase proportional to $[l(l+1)]^{1 / 2}$ in the above figures. The same applies to the one-halo term of the dark matter contribution for $l \lesssim 10^{4}$, corresponding to angular scales $\theta \simeq \pi / l \gtrsim 0.02^{\circ}$. At smaller angular scales the power spectrum is suppressed by the inner structure of the dark matter halos. We can estimate this critical scale as follows: The one-halo term is dominated by the brightest halos which correspond to the largest and nearest halos. In our fiducial scenario, the annihilating dark matter in the largest halos can emit a radio flux of $\sim 7 \times 10^{28} \mathrm{erg} \mathrm{s}^{-1} \mathrm{~Hz}^{-1}$ at $2 \mathrm{GHz}$, such that the minimum co-moving distance is $r \simeq 830 \mathrm{Mpc}, z \simeq 0.2$ if sources brighter than $0.1 \mathrm{mJy}$ are removed. The scale $r_{s}$ for the corresponding $10^{14} M_{\odot}$ halo is about $0.21 \mathrm{Mpc}$. This corresponds to a multipole $l \simeq \pi r / r_{s} \simeq 1.2 \times 10^{4}$. This simple estimation is consistent with our detailed calculation shown in Fig. 4. 


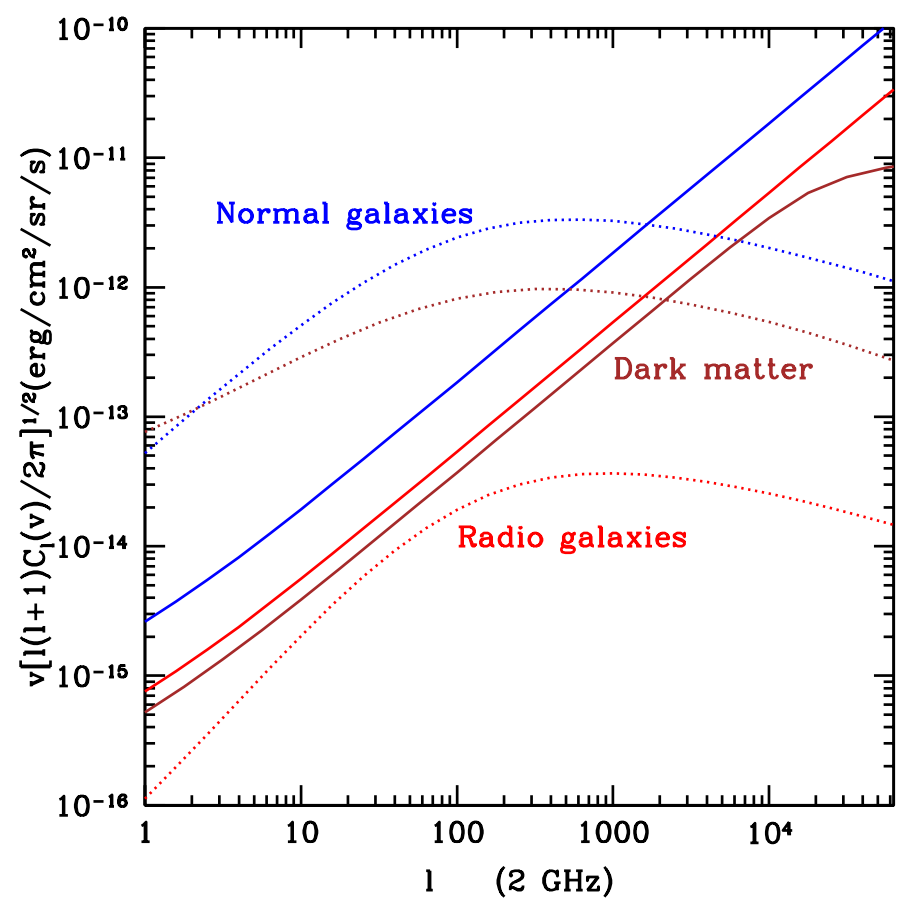

Figure 4. Angular power spectra of various components at $2 \mathrm{GHz}$. Solid lines and dotted lines represent the one-halo and two-halo terms, respectively. We assume the astrophysical sources to be point-like. The minimal dark matter halo mass is $M_{\min }=10^{6} M_{\odot}$. Sources at distances below $r_{\min }=1 \mathrm{Mpc}$, and of apparent luminosity above $S_{\text {cut }}=0.1$ mJy were removed. Color keys are as in Fig. 2 .

Figs. 4, 5, and 6 show that for radio galaxies the one-halo term is always larger than the two-halo term at all multipoles, as expected because of the high luminosity of radio galaxies. For dark matter and normal galaxies, the two-halo term dominates at small $l$. The dependence of the angular power spectrum on $l$ can potentially be used to discriminate the dark matter signal from astrophysical contributions: For $l \lesssim 3 \times 10^{3}$, the annihilation power spectrum looks significantly flatter than the signal from normal galaxies. In other words, at large angular scales, the annihilation signal has relatively more power. This can be understood as follows: After cutting bright sources, many more dim nearby annihilation sources than galaxies contribute. In addition, at large redshift the synchrotron emission from dark matter annihilation is suppressed by the increased inverse Compton scattering rate on the CMB, see Eq. (33). The twohalo term is proportional to $P_{\text {lin }}(k)$ which peaks at $\simeq 0.03 \mathrm{Mpc}^{-1}$, corresponding to $l \simeq 0.03 r(z) / \mathrm{Mpc}$. The on average smaller distance to the dark matter halos then translates into relatively more power at small $l$.

In Fig. 5, we take into account the spatial extent of the radio emission of galaxies. We assume the luminosity profiles of galaxies to be roughly proportional to the dark matter density profile which is obtained following Appendix A.2 with the halo mass is obtained from the relation between mass and bolometric luminosity [59]. As a result, for normal and radio galaxies the one-halo term starts to drop for $l \gtrsim 6000$ and $l \gtrsim 2.5 \times 10^{4}$, 


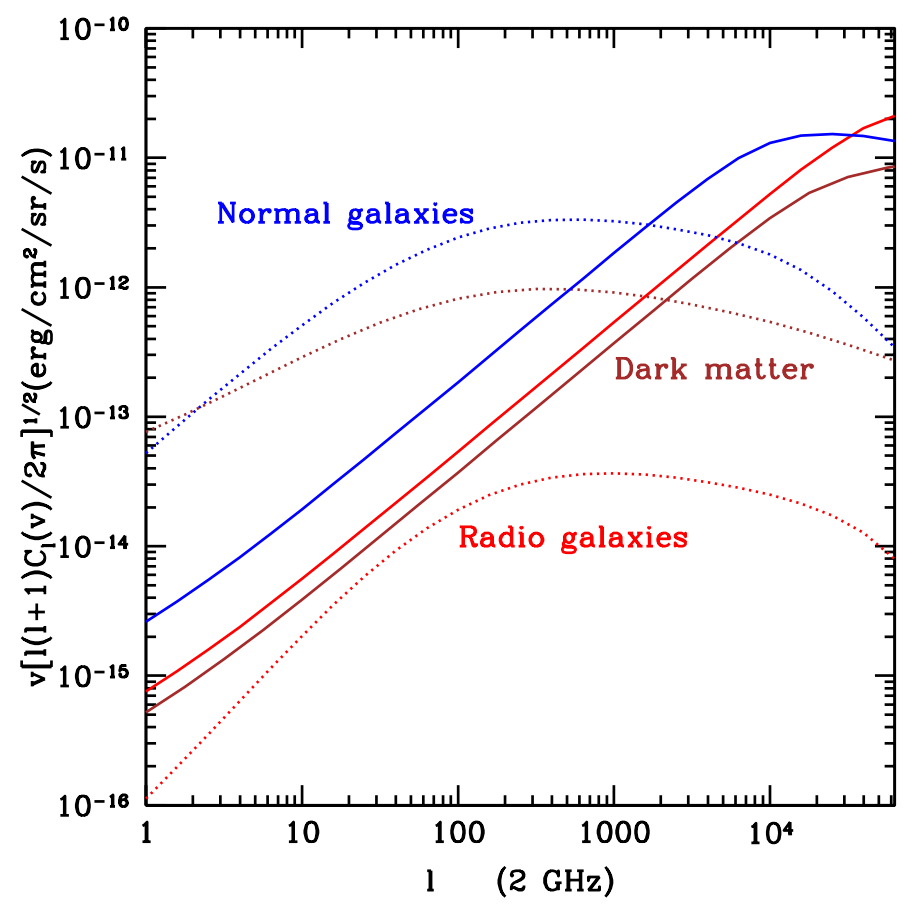

Figure 5. Same as Fig. 4, but assuming the emission profile of the astrophysical sources follows an NFW profile. Sources with luminosities above $S_{\text {cut }}=0.1 \mathrm{mJy}$ are again subtracted.

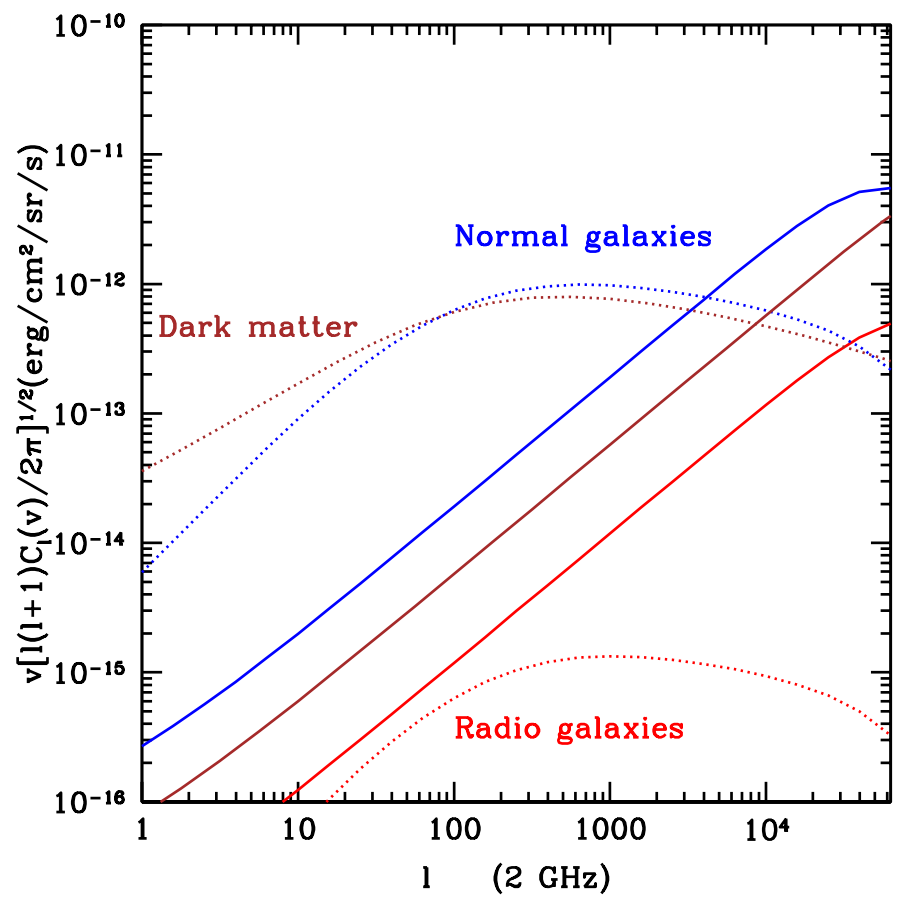

Figure 6. Same as Fig. 5, but subtracting sources above $S_{\text {cut }}=1 \mu \mathrm{Jy}$. 


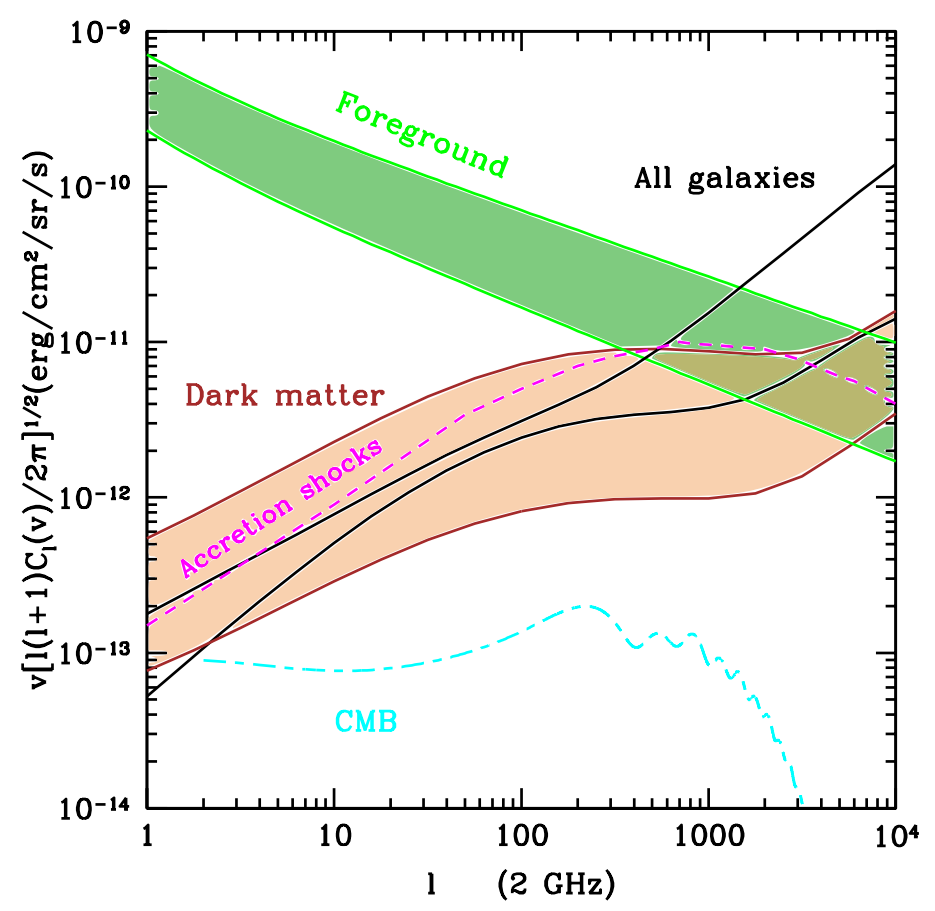

Figure 7. Angular power spectra of the radio sky at $2 \mathrm{GHz}$ compared with various estimates of the Galactic foreground at Galactic latitude $b>20^{\circ}$ (green shaded region) and the CMB (cyan curve). The brown band represents the annihilation spectrum, where the upper and lower ends correspond to $F_{\mathrm{dm}}=10$ and $F_{\mathrm{dm}}=1$, respectively, see Eq. (39), and from which halos brighter than $0.1 \mathrm{mJy}$ were removed. The blackdotted and black-solid curves represent the total signal from normal and radiogalaxies, for luminosity cuts $S_{\text {cut }}=10 \mathrm{mJy}$ and $S_{\text {cut }}=0.1 \mathrm{mJy}$, respectively. Also shown is a possible contribution from intergalactic shocks [56], normalized such that its angular power spectrum is comparable to the Galactic foreground.

respectively. Compared to the dark matter signal, the suppression thus sets in at slightly smaller $l$ for normal galaxies, but only at larger $l$ for radio galaxies. For normal galaxies this is due to the more extended emission profile which more closely follows the density as opposed to the squared density in case of dark matter. This is also reflected by the Fourier transform of the emission profiles shown in Fig. A1. For radio galaxies this effect is overcompensated by the fact that they are much brighter such that after cutting bright nearby sources, their average distance is much larger where their angular extent appears smaller.

Since future radio detectors such as the square kilometer array (SKA) [60] can reach point flux sensitivities of $\sim 1 \mu \mathrm{Jy}$, we show the power spectra of the background remaining after a corresponding luminosity cut in Fig. 6. Since the two-halo term from dark matter annihilation is insensitive to such luminosity cuts whereas the contribution from galaxies decreases rapidly, as shown in Fig. 2, the relative contribution of dark matter annihilation increases and gives rise to a flatter power spectrum at moderate $l$.

We now have to compare the cosmological background power spectra discussed so 
far with other potential contaminations. Fig. 7 compares the signals from ordinary and radio galaxies and from our fiducial dark matter scenario with the power spectra of the $\mathrm{CMB}$ and of the Galactic foreground at high Galactic latitude. The power spectrum of the Galactic foreground is not very well measured and we represent its uncertainties as a green band in Fig. 7. At high Galactic latitude below $10 \mathrm{GHz}$ local foreground fluctuations dominate over the CMB power spectrum which is why the CMB anisotropy measurements are performed above $20 \mathrm{GHz}$. Concerning annihilation signatures of dark matter with mass $m_{X} \gtrsim 100 \mathrm{GeV}$ in the angular power spectrum of the radio sky, the optimal frequency band is around $2 \mathrm{GHz}$. At higher frequencies, the synchrotron emission of electrons produced from dark matter annihilations cuts off due to Eq. (22) and the CMB signal increases. At lower frequencies, synchrotron emission by Galactic electrons dominates the power spectrum even at high Galactic latitude [61, 62, 63, 64]. Around $2 \mathrm{GHz}$, Galactic synchrotron emission always dominates, whereas free-free emission is a factor few smaller.

Also shown in Fig. 7 is a possible signal from intergalactic shocks [56]. Since its normalization is rather uncertain, we normalized it such that it is comparable to the average estimate of the Galactic foreground. The thermal SZ effect [65] is another characteristic contamination caused by hot ionized gas in galaxy clusters and filaments outside of clusters [66]. Since it dominates at small angular scales, $l \gtrsim 3000$, and at high frequencies above $30 \mathrm{GHz}$, we can neglect this effect here.

As can be seen from comparing Fig. 5 and 6 and from Fig. 7, future radio telescope arrays sensitive around $\nu \sim 2 \mathrm{GHz}$, with their higher point flux sensitivities should allow to further reduce the contribution from galaxies, whereas for $l \lesssim 6000$ the dark matter contribution is hardly changed by removing still fainter sources. This can be understood from the fact that the dark matter signal is dominated by the two-halo term which is insensitive to $S_{\text {cut }}$ for $S_{\text {cut }} \gtrsim 1 \mathrm{nJy}$, see Fig. 2. This shows that for dark matter annihilation the distribution of $l(l+1) C_{l}$ is nearly flat for $200 \lesssim l \lesssim 2000$. At smaller $l$ the power spectrum is dominated by Galactic foregrounds and at larger $l$ the one-halo term from galaxies grows rapidly. The most sensitive range $200 \lesssim l \lesssim 3000$ should be accessible to present and future radio telescopes with their high angular resolution. The SKA will have a sensitivity of about $6 \times 10^{-13} \mathrm{erg} \mathrm{cm}^{-2} \mathrm{sr}^{-1} \mathrm{~s}^{-1}$ in the units of the above figures.

We conclude that the power spectrum from dark matter annihilation tends to be flatter than other contributions because of an interplay of the following effects:

- The astrophysical signals are dominated by fewer and much brighter sources than the dark matter annihilation signal which consists of many faint sources. For $S_{\text {cut }}=0.1 \mathrm{mJy}$, the two-halo term dominates for $l \lesssim 10^{3}$ for both the signals from galaxies and from dark matter annihilation. In addition, the dark matter signal is significantly flatter in that angular range, i.e. it has relatively more power at small $l$. This is because the two-halo term is proportional to the linear power spectrum whose peak in wavenumber for the on average closer and dimmer dark matter annihilation sources translates into smaller $l$ at these luminosities. 
- For $l \gtrsim 10^{4}$, the inner spatial structure of the galaxies and dark matter halos becomes important. The inner structure tends to suppress the power spectra, but the exact angular scale at which these effects become important depends on the halo size, the profile of the emission and source luminosity cut-off.

- The various components evolve differently with the Universe expansion. For example, at high redshift inverse Compton scattering on the CMB tends to suppress synchrotron emission in dark matter halos, whereas astrophysical sources such as radio galaxies tend to be more active at $z \simeq 3$.

\subsection{Dark Matter Constraints}

We can now scale the dark matter signal to parameter values different from the fiducial scenario, by multiplying with the factor

$$
F_{\mathrm{dm}} \equiv\left(\frac{A_{b}}{10}\right)\left(\frac{Y_{e}}{10}\right)\left(\frac{\langle\sigma v\rangle}{3 \times 10^{-26} \mathrm{~cm}^{3} \mathrm{~s}^{-1}}\right)\left(\frac{100 \mathrm{GeV}}{m_{X}}\right)^{2}\left(\frac{10 \mu \mathrm{G}}{B}\right)^{1 / 2}
$$

We caution that a boost factor as high as $A_{b} \simeq 10$ has not been verified in all dark matter structure simulations and that the average magnetic field $B$ could be significantly smaller than $10 \mu \mathrm{G}$ if many small-scale subhalos contribute. However, smaller values for $A_{b}$ and $B$ partially compensate in Eq. (39) so that one could still obtain observable signatures as long as $B$ is large enough to produce emission at $\mathrm{GHz}$ frequencies, see Eq. (22).

If we choose $F_{\mathrm{dm}}=10$ (upper end of brown band in Fig. 7 ) and $S_{\text {cut }}=0.1 \mathrm{mJy}$, the annihilation spectrum dominates over other cosmological backgrounds for $100 \lesssim l \lesssim 10^{4}$ and should become distinguishable from the Galactic foreground. Note that this foreground is likely further reduced close to the Galactic poles. In this situation it should thus be possible to disentangle the rather flat power spectrum of the dark matter annihilation signal from other contributions in the range of $200 \lesssim l \lesssim 3000$. We can thus assert that radio observations are sensitive to

$$
F_{\mathrm{dm}} \gtrsim 10
$$

with some dependence on the source luminosity cut-off $S_{\text {cut }}$. Note that the dark matter signal shown in Fig. 7 does not strictly scale with $F_{\mathrm{dm}}$ because it depends on sources dimmer than $S_{\text {cut }}$, here chosen as $0.1 \mathrm{mJy}$. However, since few brighter dark matter halos contribute, the signal scales with $F_{\mathrm{dm}}$ in first approximation.

One can also compare observed radio source counts as a function of apparent point source flux with predictions for astrophysical sources and dark matter annihilation sources. This is done in Fig. 8 for the same parameters as used in Fig. 7. This establishes the constraint $F_{\mathrm{dm}} \lesssim 10$. In contrast, Fig. 7 provides dark matter signatures for future measurements but currently does not allow to put a constraint on $F_{\mathrm{dm}}$ because of the uncertainties in the Galactic foreground spectrum. Note that a future observational extension of the source count spectrum in Fig. 8 to apparent luminosities $S \lesssim \mu \mathrm{Jy}$ will provide an additional test for dark matter which predicts a shallower source count distribution than astrophysical sources. 


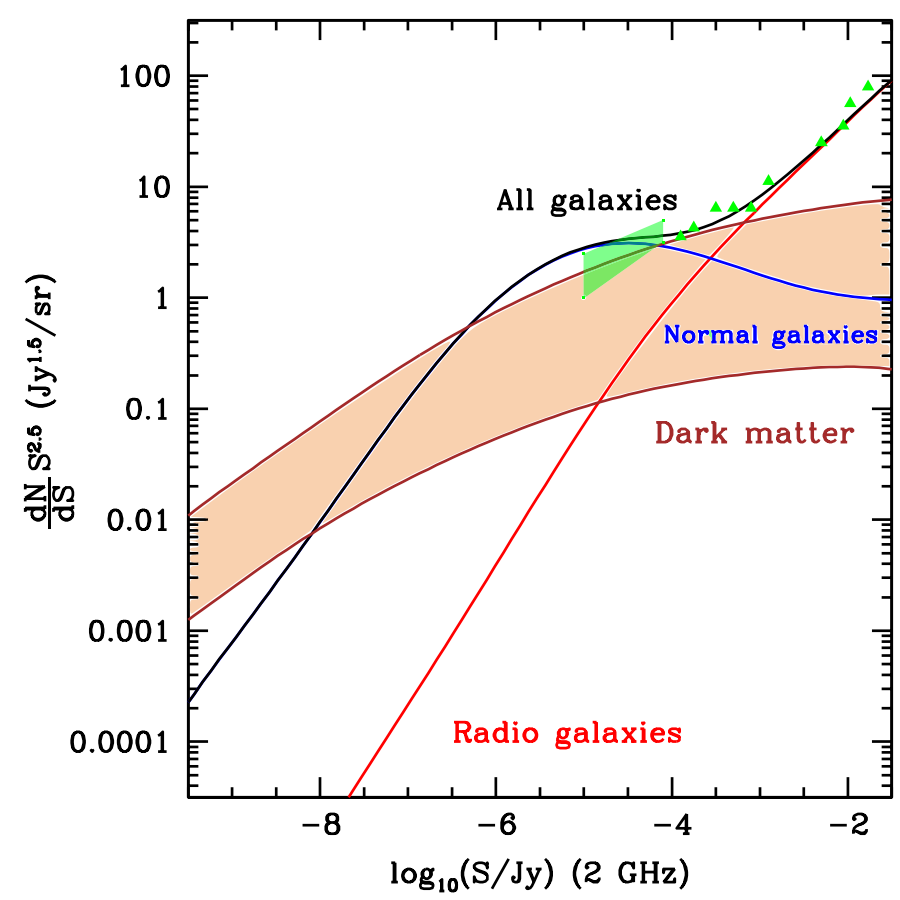

Figure 8. Observed radio source counts $(d N / d S) S^{2.5}$ as function of apparent radio flux $S$ compared with predictions for normal galaxies (blue curve), radio galaxies (red curve), and annihilations from dark matter halos (brown band, for $1 \lesssim F_{\mathrm{dm}} \lesssim 10$ ). Green shaded region and triangles are data from Ref. [67].

We have not computed the contribution from dark matter annihilations in our own Galaxy to the anisotropic radio flux in the present work. However, we know from Refs. [10, 21] that for our fiducial values for cross section and mass, at least the smooth halo component does not lead to fluxes higher than current observations from WMAP. The contribution from Galactic substructures is probably more model dependent than our cosmological flux which apart from an overall boost factor depends only on the host halo distribution and effectively averages over a much larger ensemble of halos. This can also be seen from Ref. [68] where the predictions of the $\gamma$-ray flux from Galactic dark matter annihilations varied over orders of magnitude.

\section{Conclusions}

Many different indirect detection signatures have been investigated to constrain the parameter space for dark matter $[8,12,69,70,71,72,73]$. In the present paper we have calculated intensity and angular power spectrum of the cosmological background of synchrotron emission from the electrons and positrons produced in annihilations of cold dark matter. The resulting radio background around $\simeq 2 \mathrm{GHz}$ and its angular power spectrum for multipoles $200 \lesssim l \lesssim 3000$ has comparable or better sensitivity to dark matter annihilation cross sections than other signatures. Furthermore, a comparison of observed radio source counts with predictions for dark matter annihilation results 
in the constraint $F_{\mathrm{dm}} \lesssim 10$ for the parameter defined in Eq. (39). Under reasonable assumptions on dark matter clustering and magnetic fields in the halo environment, the range of annihilation cross sections corresponding to the constraint $F_{\mathrm{dm}} \lesssim 10$ is comparable to constraints from synchrotron emission in an NFW profile [10, 21]. Galactic $\gamma$-ray constraints derived under similar assumptions [74] are also comparable. The sensitivity of our signal is considerably better than conservative limits based on annihilation into neutrinos [14], and comparable to limits on annihilation into $\gamma$-rays from diffuse cosmological emission [13].

Sensitivities to values of order ten for the parameter $F_{d m}$ defined in Eq. (39)

are interesting for non-thermal dark matter whose annihilation cross sections can be larger than our fiducial value $\langle\sigma v\rangle=3 \times 10^{-26} \mathrm{~cm}^{3} \mathrm{~s}^{-1}$, the cross section required for thermal dark matter. An example for a non-thermal dark matter candidate with large cross section is the wino LSP occurring in supersymmetric theories with anomaly mediation $[75,76]$. We believe that radio observations in particular with future instruments such as SKA can provide valuable information on dark matter.

\section{Acknowledgements}

We acknowledge partial support by the DFG (Germany) under grants SFB-676 and GK 602, and by the European Union under the ILIAS project (contract No. RII3-CT-2004506222). L.Z. would like to thank Shin'ichiro Ando, Yanchuan Cai and Yan Qu for valuable discussions.

\section{Appendix A. Cosmological Dark Matter Distribution}

\section{Appendix A.1. Power Spectrum and Halo Mass Function}

The mass function of the halo distribution is derived from the Press-Schechter formalism [26]. In this approach fluctuations in the linear density field with $\delta>\delta_{\mathrm{c}}$ decouple from the local Hubble expansion of the universe and collapse to form nonlinear structures. The fraction of the volume that has collapsed is predicted to be

$$
f_{\text {coll }}(M(R), z)=\frac{2}{\sqrt{2 \pi} \sigma(R, z)} \int_{\delta_{\mathrm{c}}}^{\infty} d \delta e^{-\delta^{2} / 2 \sigma^{2}(R, z)},
$$

where $R$ is the co-moving radius over which the density field has been smoothed, which is related to the halo mass by $M(R)=\rho_{m} 4 \pi R^{3} / 3$ with $\rho_{m}$ the co-moving matter density of the universe. The number density of halos is then found to be given by $[26,27]$

$$
\frac{d n(M, z)}{d M}=-\frac{\rho_{m}}{M} \frac{d f_{\mathrm{coll}}(M(R), z)}{d M}=\frac{\rho_{m}}{M} f(\nu) \frac{d \nu}{d M},
$$

where

$$
f(\nu) \equiv \sqrt{\frac{2 A^{2} a^{2}}{\pi}}\left[1+\left(a \nu^{2}\right)^{-p}\right] e^{-\frac{a \nu^{2}}{2}} .
$$


Here

$$
\nu(M, z) \equiv \frac{\delta_{\mathrm{c}}(z)}{\sigma(M, z)}
$$

and

$$
\delta_{\mathrm{c}}(z) \simeq 1.686
$$

is the critical density required for spherical collapse at a redshift $z$ in an Einstein-de Sitter space. The variance in the density field smoothed with a top-hat filter of radius $R=\left(3 M / 4 \pi \rho_{m}\right)^{\frac{1}{3}}$ is

$$
\sigma^{2}(M, z)=G^{2}(z) \int \frac{d k}{k} \frac{k^{3} P_{\operatorname{lin}}(k)}{2 \pi^{2}}|W(k R)|^{2},
$$

where

$$
W(x)=\frac{3}{x^{3}}[\sin (x)-x \cos (x)]
$$

$P_{\text {lin }}(k)$ is the linear matter power spectrum, and

$$
G(z)=\frac{H(z) \int_{z}^{\infty} d z^{\prime}\left(1+z^{\prime}\right)\left[H\left(z^{\prime}\right)\right]^{-3}}{H_{0} \int_{0}^{\infty} d z^{\prime}\left(1+z^{\prime}\right)\left[H\left(z^{\prime}\right)\right]^{-3}}
$$

is the growth factor with linear perturbation theory, often also denoted by $D(z)$. In Eq. (A.3) A, p, and $a$ are constants, with the canonical Press-Schechter (PS) and Sheth-Tormen (ST) mass functions corresponding to the parameters $(p=0, a=1)$ and ( $p=0.3, a=0.707$ ), respectively. The normalization $A$ is determined by requiring mass conservation such that

$$
\frac{1}{\rho_{m}} \int_{0}^{\infty} d M M \frac{d n}{d M}=\int_{0}^{\infty} d \nu f(\nu)=1
$$

For PS $A=1$ and for ST $A=0.3222$.

The primordial power spectrum $P(k) \propto A_{s} k^{n_{s}}$ can be modified by the content and evolution of different matter components of the Universe due to the perturbations that enter the horizon at different epochs. This allows one to relate the linear power spectrum to the primordial power spectrum through a transfer function $T(k)$ via

$$
P_{\text {lin }}(k, z)=D^{2}(z) P_{\operatorname{lin}}(k, z=0)=D^{2}(z) A_{s}(k \cdot \mathrm{Mpc})^{n_{s}} T^{2}(k)
$$

Fitting formula for an adiabatic CDM model give [77]

$$
T_{\mathrm{CDM}}(q)=\frac{\ln (1+2.34 q)}{2.34 q}\left[1+3.89 q+(16.1 q)^{2}+(5.46 q)^{3}+(6.71 q)^{4}\right]^{-1 / 4}(\mathrm{~A}
$$

where $q=k \cdot \operatorname{Mpc} /(h \Gamma)$ and $\left.\Gamma=\Omega_{m} h \exp \left[\Omega_{b}(1+\sqrt{(2 h}) / \Omega_{m}\right)\right]$. One usually uses the rms fluctuation on an $8 h^{-1} \mathrm{Mpc}$ scale to normalize the amplitude of the present power spectrum. From WMAP 5 year data, we adopt $n_{s}=0.96[1,2]$, and $A_{s}=1.4 \times 10^{7}$. Following Ref. [78], we may furthermore write the linear growth factor as

$$
D(z)=\frac{1}{1+z} \frac{g(z)}{g(0)}
$$

where an approximate expressionfor $g(z)$ is

$$
g(z)=\frac{5 / 2 \Omega_{m}(z)}{\Omega_{m}(z)^{4 / 7}-\Omega_{\Lambda}(z)+\left(1+\Omega_{m}(z) / 2\right)\left(1+\Omega_{\Lambda}(z) / 70\right)} .
$$


Appendix A.2. Dark Matter Density Profile

The halo mass function has to be supplemented by the dark matter density profile. For the dark matter profile within each halo we use an NFW profile [28],

$$
\rho_{h}(\mathbf{r})=\frac{\delta_{c h} \rho_{m}(1+z)^{3}}{r / r_{s}\left(1+r / r_{s}\right)^{2}}
$$

where $r_{s}$ is a characteristic radius. Within the context of the spherical collapse model, the outer extent of the cluster is taken to be the virial radius

$$
r_{v}=\left[\frac{3 M}{4 \pi \rho_{m}(1+z)^{3} \Delta_{c}(z)}\right]^{\frac{1}{3}}
$$

where $\rho_{m}(1+z)^{3}$ is the average physical background matter density of the universe at redshift $z$, and

$$
\Delta_{c}(z) \simeq 18 \pi^{2}\left[1+\frac{88}{215}\left(\frac{1-\Omega_{m}}{\Omega_{m}(1+z)^{3}}\right)^{\frac{86}{95}}\right]
$$

is the overdensity of the halo relative to the background density [79]. The ratio of the virial radius to the scale radius is called the concentration parameter $c \equiv r_{v} / r_{s}$. A combination of the definitions of virial mass and density profile gives

$$
\delta_{c h}=\frac{\Delta_{c}(z)}{3} \frac{c^{3}}{\ln (1+c)-c /(1+c)}
$$

Together, $c$ and $M$ completely determine the dark matter distribution of a given halo. Note that an NFW profile is a conservative assumption compared to steeper profiles that have been proposed, for example the Moore profile [80], which would consequently lead to a larger dark matter signal.

\section{Appendix A.3. Concentration Distribution}

It is still uncertain how the concentration parameter depends on mass and redshift. In

our paper we consider a realistic value of $c\left(M=10^{-6} M_{\odot}, z=0\right) \simeq 70$. Extrapolating to the low mass range, we use [34]

$$
c(M, z)=4 \frac{1+z_{c}}{1+z},
$$

where the collapse redshift $z_{c}$ is implicitly given by the relation $M_{*}\left(z_{c}\right)=0.01 M$, where $M_{*}(z)$ is the mass scale at which $\sigma\left(M_{*}, z\right)=\delta_{c}$. In a less conservative parametrization motivated by numerical simulations $c$ would follow a log-normal distribution with standard deviation $\sigma_{c}=0.18$,

$$
\mathcal{P}(\ln c \mid M, z)=\frac{1}{\sqrt{2 \pi} \sigma_{c}} \exp \left(-\frac{[\ln c-\ln \bar{c}(M, z)]^{2}}{2 \sigma_{c}^{2}}\right),
$$

where the mean concentration parameter $\bar{c}$ is related to the halo mass via [36]

$$
\bar{c}(M, z)=\frac{c_{0}}{1+z}\left[\frac{M}{M_{*}(z=0)}\right]^{-\alpha_{c}},
$$


where $c_{0}$ and $\alpha_{c}$ are constants whose numerical values [34] are typically chosen to be $c_{0}=9$ and $\alpha_{c}=0.13$. However, application of this parameterization to low-mass halos and to high redshift give values inconsistent with some simulations [29]. A third parameterization [35] uses

$$
\bar{c}(M, z)=a(z)\left[\frac{M}{M_{*}(z)}\right]^{b(z)},
$$

with $a(z)=10.3(1+z)^{-0.3}$, and $b(z)=0.24(1+z)^{-0.3}$. The parametrization Eqs. (A.19), (A.20) give comparable dark matter signals, whereas the parametrization Eqs. (A.21) would lead to signals about a factor 20 higher than our conservative calculation.

\section{Appendix A.4. Bias}

For the linear dark matter halo bias $b(M, z)$ appearing in Eq. (6) we adopt [81]

$$
b(M, z)=1+\frac{\nu^{2}(M, z)}{\delta_{c} D(z)},
$$

whereas for the galaxy bias we simply use unity.

\section{Appendix A.5. Bolometric Luminosity}

In order to compute the Fourier transform of galaxy density profiles, we first need the galaxy mass. For normal galaxies we use the relation [59]

$$
M=14 \frac{L_{60} \nu_{60}}{L_{\text {sun }}} \frac{\Omega_{m}}{\Omega_{b}} M_{\odot},
$$

where $L_{60}$ is the luminosity at 60 microns, and $\nu_{60} \sim 5000 \mathrm{GHz}$ is its frequency, and $L_{\odot} \sim 3.9 \times 10^{33} \mathrm{erg} / \mathrm{s}$ is the solar bolometric luminosity. For radio galaxies we simply adopt their typical mass of about $10^{12} M_{\odot}$ to estimate the Fourier transform of the their density profile.

\section{Appendix A.6. Fourier Transforms}

The Fourier transform of the spherically symmetric NFW profile of mass M can be written as

$$
\mathcal{F}_{\rho_{h}}(k, M)=\int_{0}^{r_{v}} \rho_{h}(M, r) \frac{\sin (k r)}{k r} 4 \pi r^{2} d r,
$$

and analogously for $\mathcal{F}_{\rho_{h}^{2}}(k, M)$. For the purpose of plotting these Fourier transforms, see Fig. A1, it is convenient to renormalize them to unity for $k \rightarrow$ 0 by introducing the new functions $y_{1}(k, M)=\mathcal{F}_{\rho_{h}}(k, M) / M$ and $y_{2}(k, M)=$ $\mathcal{F}_{\rho_{h}^{2}}(k, M) / \int d V_{h} \rho_{h}^{2}(\mathbf{r})$. We then have $y_{i}(0, M)=1$, and $y_{i}(k>0, M)<1$ for $i \stackrel{2}{=} 1,2$. For the NFW density profile, $\int d V_{h} \rho_{h}^{2}(\mathbf{r})=f_{c} M \rho_{m} \Delta_{c}(z)$, where $f_{c}=$ $\left(c^{3} / 9\right)\left[1-(1+c)^{-3}\right] /[\log (1+c)-c /(1+c)]^{2}$, and $\Delta(z) \sim 200$.

For the NFW profile, the mass of the halo within radius $r$ increases $\propto r^{2}$ for $r \lesssim r_{s}$, and then increase logarithmically for $r_{s} \lesssim r \lesssim r_{v}$ where $\rho_{h}(r) \propto r^{-3}$. Therefore, the 


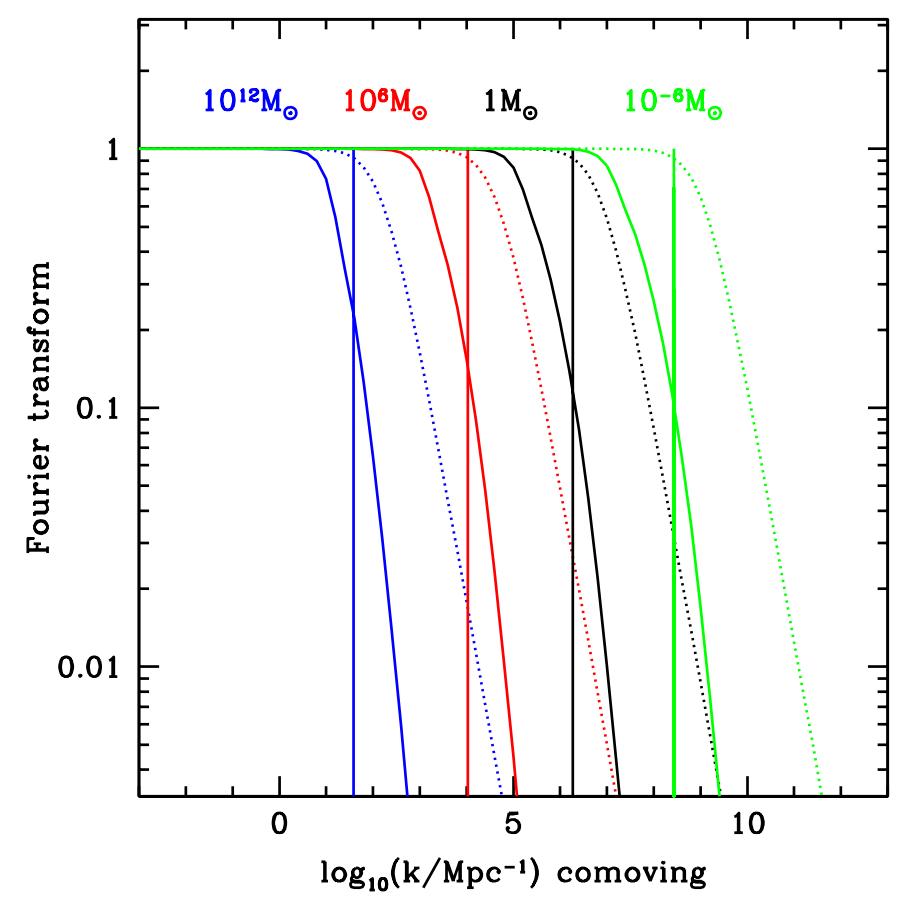

Figure A1. The normalized Fourier transforms $y_{1}(k, M)$ (solid lines) and $y_{2}(k, M)$ (dotted lines) of $\rho_{h}$ and $\rho_{h^{2}}$, respectively, as functions of co-moving wavenumber $k$. The vertical lines denote the scale $k=1 / r_{s}(M)$.

dominant contribution to the halo mass comes from $r \lesssim r_{s}$. Similarly, the annihilation signal is produced mainly within $r \lesssim r_{s}$, increasing there $\propto r$, but increases only $\propto r_{s}^{-3}-r^{-3}$ for $r_{s} \lesssim r \lesssim r_{v}$.

Fig. A1 shows that for $k r_{s} \ll 1$ we have $y_{1,2} \simeq 1$, whereas for $k r_{s} \gg 1$ one has $y_{2}(k, M) \propto k^{-1}$, and $y_{1}(k, M) \propto k^{-2}$.

\section{Appendix A.7. Foregrounds}

The radio intensity $I_{\nu}$ at a given frequency $\nu$ can be expressed in terms of antenna temperature $T_{A}(\nu)$ via $I_{\nu}=2 \nu^{2} k_{\mathrm{B}} T_{A}(\nu) / c_{0}^{2}$, where $c_{0}$ is the speed of light. Alternatively, $I_{\nu}$ can be written in terms of the thermodynamic temperature as the temperature of a blackbody with the given intensity at frequency $\nu$, thus $I_{\nu}=2 \nu^{3} /\left(e^{x}-1\right)$, where $x \equiv h \nu / k_{\mathrm{B}} T$ with $h$ the Planck constant. Thus, for power law spectra $I_{\nu} \propto \nu^{\alpha}, T_{A} \propto \nu^{\alpha-2}$. In general, the CMB is expressed in terms of thermodynamic temperature $T$, while Galactic and extragalactic foregrounds are expressed in term of antenna temperature. Thermodynamic and antenna temperature are then related by $T=T_{A}\left(e^{x}-1\right) / x$, and their fluctuations by $\Delta T=\Delta T_{A}\left(e^{x}-1\right)^{2} /\left(x^{2} e^{x}\right)$. For the CMB, $x=h \nu /\left(k_{\mathrm{B}} T_{\mathrm{CMB}}\right) \simeq \nu /(56.8 \mathrm{GHz})$ with the $\mathrm{CMB}$ temperature $T_{\mathrm{CMB}}=2.725 \mathrm{~K}[58]$. Since we consider frequencies $\nu \lesssim 10 \mathrm{GHz}$ in the present paper, $x \ll 1$ and thus $T \simeq T_{A}$ and $\Delta T \simeq \Delta T_{A}$. 
From the definition of $T_{A}$ we get

$I_{\nu}=3.06 \times 10^{-25}\left(\frac{\nu}{\mathrm{GHz}}\right)^{2}\left(\frac{T_{A}}{\mu \mathrm{K}}\right) \mathrm{erg} \mathrm{cm}^{-2} \mathrm{~s}^{-1} \mathrm{~Hz}^{-1} \mathrm{sr}^{-1}$.

Since for $I_{\nu} \propto \nu^{\alpha}$ the power spectrum $C_{l}^{I_{\nu}}$ of $I_{\nu}$ at frequency $\nu$ scales as $\nu^{2 \alpha}$, we can express it in terms of the power spectrum $C^{T_{A}}\left(\nu^{\prime}\right)$ of the antenna temperature $T_{A}$ at frequency $\nu^{\prime}$ via

$\sqrt{C_{l}^{I_{\nu}}(\nu)}=3.06 \times 10^{-25}\left(\frac{\nu}{\nu^{\prime}}\right)^{\alpha-2} \sqrt{\frac{C_{l}^{T_{A}}\left(\nu^{\prime}\right)}{\mu \mathrm{K}^{2}}}\left(\frac{\nu}{\mathrm{GHz}}\right)^{2} \operatorname{erg~cm}^{-2} \mathrm{~s}^{-1} \mathrm{~Hz}^{-1} \mathrm{sr}^{-1}$.

Here, $\alpha=-0.9$ for synchrotron emission and -0.15 for free-free emissions, respectively [62]. For the normalization and the dependence on $l$, we adopted the best-fit model from observations at $2.3 \mathrm{GHz}$ [63]. These parametrizations have been used in Fig. 7.

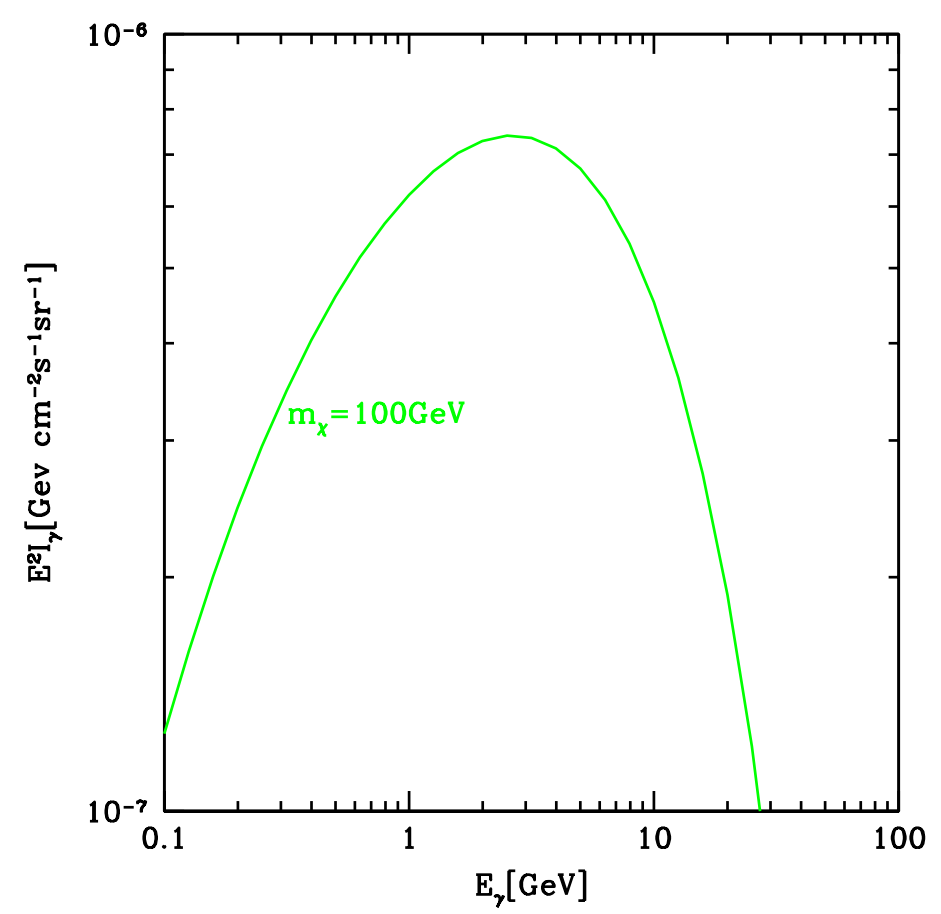

Figure A2. Diffuse energy spectrum of $\gamma$-rays from dark matter annihilation for our fiducial scenario, with $M_{\min }=10^{-6} M_{\odot}$. This is consistent with Fig. 1 in Ref. [8] within about $10 \%$.

Appendix A.8. Diffuse Flux and Power Spectrum of $\gamma$-rays

We can use our approach also to compute diffuse energy spectra and angular power spectra of $\gamma$-rays from dark matter annihilation. For neutralinos the spectrum of $\gamma$-rays of energy $E_{\gamma}$ per annihilation can be parameterized by the simple expression [4]

$$
\frac{d N_{\gamma}}{d E}\left(E_{\gamma}\right) \simeq \frac{0.73}{m_{X}} \frac{e^{-7.776 E_{\gamma} / m_{X}}}{\left(E_{\gamma} / m_{X}\right)^{1.5}+0.00014} .
$$




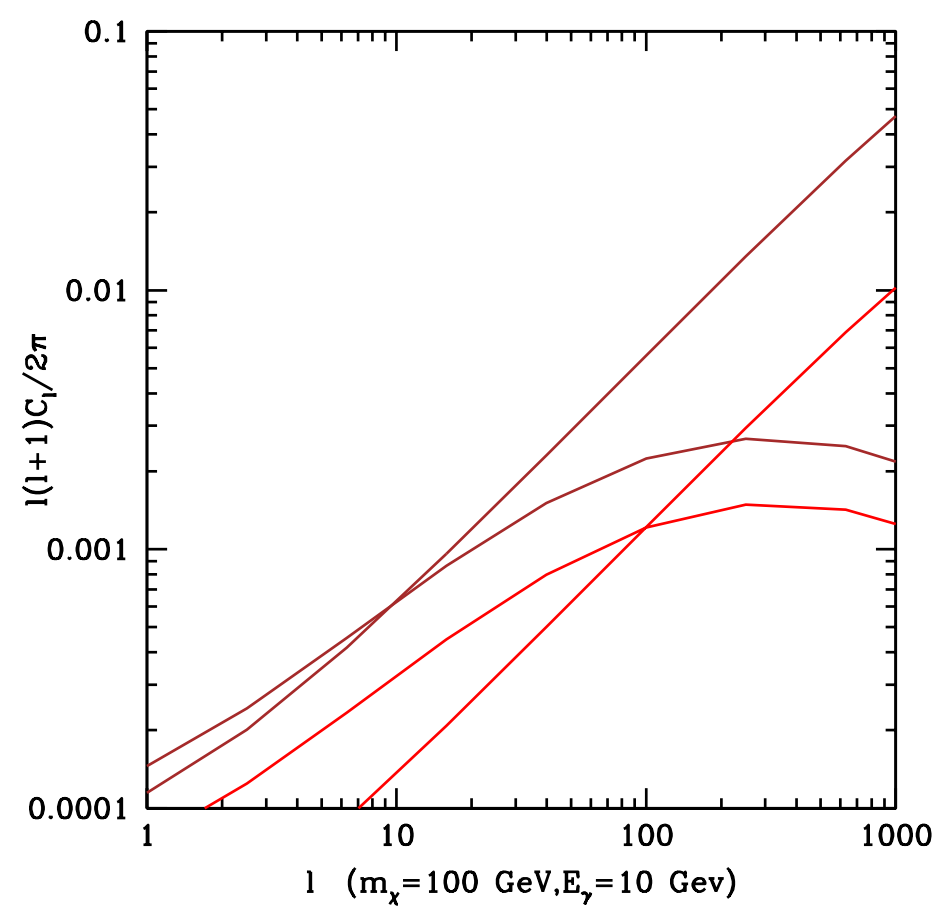

Figure A3. Angular power spectrum of $10 \mathrm{GeV} \gamma$-rays from dark matter annihilation in our fiducial scenario. Brown lines are for minimal halo mass $M_{\min }=10^{6} M_{\odot}$, whereas red lines are for $M_{\min }=10^{-6} M_{\odot}$. The higher and lower curves at high $l$ denote the one-halo and two-halo terms, respectively. Note that $C_{l}$ is now normalized to the total intensity squared, $I_{\nu}^{2}$. This is consistent with Fig. 6 in Ref. [8] within a factor $\simeq 2$.

To reasonably match the EGRET data [53], and compare with the results in Ref. [8], we multiply the predicted average $\gamma$-ray intensity spectrum by the boost factor $A_{b} \sim 240$ due to substructure within the host halos. Note that this boost factor is much more extreme than $A_{b} \simeq 10$ assumed in the present work. We then have

$$
w\left(E_{\gamma}, z\right)=\frac{\langle\sigma v\rangle}{8 \pi}\left(\frac{\Omega_{m}}{m_{X}}\right)^{2}(1+z)^{3} E_{\gamma} \frac{d N_{\gamma}\left(E_{\gamma}, z\right)}{d E_{\gamma}}
$$

for the weight function in Eq. (28). For our fiducial dark matter scenario we then obtain Fig. A2 for the solid angle averaged energy spectrum and Fig. A3 for the angular power spectrum at $\gamma$-ray energy $E_{\gamma}=10 \mathrm{GeV}$. Note that if fainter sources were included, corresponding to smaller minimal halo mass $M_{\min }$, the relative fluctuations normalized to $I_{\nu}^{2}$, and especially the one-halo Poisson term, would decrease.

The diffuse energy spectrum is consistent with the results in Ref. [8] within about $10 \%$. In addition, the angular power spectrum at $\gamma$-ray energy $10 \mathrm{GeV}$ is also consistent with the results in Ref. [8] within a factor two. This serves as an important cross-check of our results with independent calculations. 


\section{References}

[1] E. Komatsu et al. [WMAP Collaboration], arXiv:0803.0547 [astro-ph].

[2] J. Dunkley et al. [WMAP Collaboration], arXiv:0803.0586 [astro-ph].

[3] G. Bertone, D. Hooper and J. Silk, Phys. Rept. 405, 279 (2005) [arXiv:hep-ph/0404175].

[4] L. Bergstrom, J. Edsjo and P. Ullio, annihilations," Phys. Rev. Lett. 87, 251301 (2001) [arXiv:astro-ph/0105048].

[5] P. Ullio, L. Bergstrom, J. Edsjo and C. G. Lacey, look," Phys. Rev. D 66, 123502 (2002) [arXiv:astro-ph/0207125].

[6] J. E. Taylor and J. Silk, Mon. Not. Roy. Astron. Soc. 339, 505 (2003) [arXiv:astro-ph/0207299].

[7] D. Elsaesser and K. Mannheim, background," Phys. Rev. Lett. 94, 171302 (2005) [arXiv:astro$\mathrm{ph} / 0405235]$.

[8] S. Ando and E. Komatsu, Phys. Rev. D 73, 023521 (2006) [arXiv:astro-ph/0512217].

[9] S. Ando, E. Komatsu, T. Narumoto and T. Totani, Phys. Rev. D 75, 063519 (2007) [arXiv:astro$\mathrm{ph} / 0612467]$.

[10] D. Hooper, G. Zaharijas, D. P. Finkbeiner and G. Dobler, WMAP Haze," arXiv:0709.3114 [astro$\mathrm{ph}$.

[11] A. Cuoco, J. Brandbyge, S. Hannestad, T. Haugboelle and G. Miele, Phys. Rev. D 77, 123518 (2008) [arXiv:0710.4136 [astro-ph]].

[12] S. Ando, E. Komatsu, T. Narumoto and T. Totani, Mon. Not. Roy. Astron. Soc. 376, 1635 (2007) [arXiv:astro-ph/0610155].

[13] G. D. Mack, T. D. Jacques, J. F. Beacom, N. F. Bell and H. Yuksel, arXiv:0803.0157 [astro-ph].

[14] H. Yuksel, S. Horiuchi, J. F. Beacom and S. Ando, Phys. Rev. D 76, 123506 (2007) [arXiv:0707.0196 [astro-ph]].

[15] L. Bergstrom, J. Edsjo, M. Gustafsson and P. Salati, JCAP 0605, 006 (2006) [arXiv:astro$\mathrm{ph} / 0602632]$.

[16] T. Bringmann and P. Salati, Phys. Rev. D 75, 083006 (2007) [arXiv:astro-ph/0612514].

[17] T. Delahaye, R. Lineros, F. Donato, N. Fornengo and P. Salati, Phys. Rev. D 77, 063527 (2008) [arXiv:0712.2312 [astro-ph]].

[18] G. Bertone, G. Sigl and J. Silk, Mon. Not. Roy. Astron. Soc. 326, 799 (2001) [arXiv:astro$\mathrm{ph} / 0101134]$.

[19] G. Bertone, G. Sigl and J. Silk, Mon. Not. Roy. Astron. Soc. 337, 98 (2002) [arXiv:astro$\mathrm{ph} / 0203488]$.

[20] D. Hooper, Phys. Rev. D 77, 123523 (2008) [arXiv:0801.4378 [hep-ph]].

[21] P. Grajek, G. Kane, D. J. Phalen, A. Pierce and S. Watson, arXiv:0807.1508 [hep-ph].

[22] M. Regis and P. Ullio, arXiv:0802.0234 [hep-ph].

[23] R. J. Protheroe and P. L. Biermann, Astropart. Phys. 6, 45 (1996) [Erratum-ibid. 7, 181 (1997)] [arXiv:astro-ph/9605119].

[24] Rybicki G.B., Lightman A.P., Radiative Processes in Astrophysics, 1979, John Wiley \& Sons.

[25] Kaiser, N., Astrophys. J. 388, 272 (1992)

[26] W. H. Press and P. Schechter, Astrophys. J. 187, 425 (1974).

[27] R. K. Sheth and G. Tormen, Mon. Not. Roy. Astron. Soc. 308, 119 (1999) [arXiv:astro$\mathrm{ph} / 9901122]$.

[28] J. F. Navarro, C. S. Frenk and S. D. M. White, Astrophys. J. 462, 563 (1996) [arXiv:astro$\mathrm{ph} / 9508025]$

[29] J. Diemand, B. Moore and J. Stadel, Nature 433 (2005) 389 [arXiv:astro-ph/0501589].

[30] S. Hofmann, D. J. Schwarz and H. Stoecker, Phys. Rev. D 64, 083507 (2001).

[31] X. l. Chen, M. Kamionkowski and X. m. Zhang, Phys. Rev. D 64, 021302 (2001).

[32] A. M. Green, S. Hofmann and D. J. Schwarz, JCAP 0508, 003 (2005).

[33] P. P. Kronberg, Rept. Prog. Phys. 57, 325 (1994).

[34] J. S. Bullock et al., Mon. Not. Roy. Astron. Soc. 321, 559 (2001) [arXiv:astro-ph/9908159]. 
[35] U. Seljak, Mon. Not. Roy. Astron. Soc. 318, 203 (2000) [arXiv:astro-ph/0001493].

[36] A. Cooray and R. K. Sheth, Phys. Rept. 372, 1 (2002) [arXiv:astro-ph/0206508].

[37] G. Tormen, A. Diaferio and D. Syer, Mon. Not. Roy. Astron. Soc. 299, 728 (1998).

[38] A. A. Klypin, S. Gottlober and A. V. Kravtsov, Astrophys. J. 516, 530 (1999).

[39] B. Moore, S. Ghigna, F. Governato, G. Lake, T. Quinn, J. Stadel and P. Tozzi, Astrophys. J. 524 (1999) L19.

[40] S. Ghigna, B. Moore, F. Governato, G. Lake, T. Quinn and J. Stadel, Astrophys. J. 544, 616 (2000).

[41] V. Springel, S. D. M. White, G. Tormen and G. Kauffmann, Mon. Not. Roy. Astron. Soc. 328, 726 (2001).

[42] A. R. Zentner and J. S. Bullock, Astrophys. J. 598, 49 (2003).

[43] G. De Lucia et al., Mon. Not. Roy. Astron. Soc. 348, 333 (2004).

[44] A. Helmi, S. D. M. White and V. Springel, Phys. Rev. D 66, 063502 (2002).

[45] L. Gao, S. D. M. White, A. Jenkins, F. Stoehr and V. Springel, Mon. Not. Roy. Astron. Soc. 355 (2004) 819.

[46] L. Shaw, J. Weller, J. P. Ostriker and P. Bode, Astrophys. J. 646, 815 (2006).

[47] J. Diemand, M. Kuhlen, P. Madau, M. Zemp, B. Moore, D. Potter and J. Stadel, arXiv:0805.1244 [astro-ph].

[48] X. J. Bi, Nucl. Phys. B 741, 83 (2006) [arXiv:astro-ph/0510714].

[49] Q. Yuan and X. J. Bi, JCAP 0705, 001 (2007) [arXiv:astro-ph/0611872].

[50] J. Lavalle, Q. Yuan, D. Maurin and X. J. Bi, arXiv:0709.3634 [astro-ph].

[51] S. W. Barwick et al. [HEAT Collaboration], Astrophys. J. 482, L191 (1997) [arXiv:astro$\mathrm{ph} / 9703192]$.

[52] S. Coutu et al., Prepared for 27th International Cosmic Ray Conference (ICRC 2001), Hamburg, Germany, 7-15 Aug 2001

[53] A. W. Strong, I. V. Moskalenko and O. Reimer, Astrophys. J. 613, 956 (2004) [arXiv:astro$\mathrm{ph} / 0405441]$.

[54] P. Sreekumar et al. [EGRET Collaboration], Astrophys. J. 494, 523 (1998) [arXiv:astro$\mathrm{ph} / 9709257]$.

[55] E. Waxman and A. Loeb, Astrophys. J. 545, L11 (2000) [arXiv:astro-ph/0007049].

[56] U. Keshet, E. Waxman and A. Loeb, Astrophys. J. 617, 281 (2004) [arXiv:astro-ph/0402320].

[57] G. F. Smoot, arXiv:astro-ph/9902201.

[58] J. C. Mather, D. J. Fixsen, R. A. Shafer, C. Mosier and D. T. Wilkinson, Astrophys. J. 512, 511 (1999) [arXiv:astro-ph/9810373].

[59] L. Spinoglio, M. A. Malkan, B. Rush, L. Carrasco and E. Recillas-Cruz, Astrophys. J. 453, 616 (1995) [arXiv:astro-ph/9506139].

[60] C. A. Jackson, New Astron. Rev. 48, 1187 (2004) [arXiv:astro-ph/0409180].

[61] M. Tegmark and G. Efstathiou, arXiv:astro-ph/9507009.

[62] M. Tegmark, D. J. Eisenstein, W. Hu and A. de Oliveira-Costa, Astrophys. J. 530, 133 (2000) [arXiv:astro-ph/9905257].

[63] G. Giardino, A. J. Banday, P. Fosalba, K. M. Gorski, J. L. Jonas, W. O'Mullane and J. Tauber, arXiv:astro-ph/0103233.

[64] L. La Porta, C. Burigana, W. Reich and P. Reich, arXiv:0801.0547 [Unknown].

[65] R. A. Sunyaev and Y. B. Zeldovich, Astrophys. Space Sci. 7, 3 (1970).

[66] Persi. F. M., Cen, R., Ostriker, J. P., Astrophys. J. 442, 1 (1995)

[67] J. J. Condon, Ap. J. 338, 13 (1989).

[68] J. M. Siegal-Gaskins, arXiv:0807.1328 [astro-ph].

[69] X. L. Chen and M. Kamionkowski, Phys. Rev. D 70, 043502 (2004) [arXiv:astro-ph/0310473].

[70] L. Zhang, X. L. Chen, Y. A. Lei and Z. G. Si, Phys. Rev. D 74, 103519 (2006) [arXiv:astro$\mathrm{ph} / 0603425]$.

[71] L. Zhang, X. Chen, M. Kamionkowski, Z. g. Si and Z. Zheng, Phys. Rev. D 76, 061301 (2007) 
[arXiv:0704.2444 [astro-ph]].

[72] Z. Myers and A. Nusser, arXiv:0710.0135 [astro-ph].

[73] N. Padmanabhan and D. P. Finkbeiner, Phys. Rev. D 72, 023508 (2005) [arXiv:astro-ph/0503486].

[74] S. Dodelson, D. Hooper and P. D. Serpico, Phys. Rev. D 77, 063512 (2008) [arXiv:0711.4621 [astro-ph]].

[75] L. Randall and R. Sundrum, Nucl. Phys. B 557, 79 (1999) [arXiv:hep-th/9810155].

[76] G. F. Giudice, M. A. Luty, H. Murayama and R. Rattazzi, JHEP 9812, 027 (1998) [arXiv:hep$\mathrm{ph} / 9810442]$.

[77] J. M. Bardeen, J. R. Bond, N. Kaiser and A. S. Szalay, Astrophys. J. 304 (1986) 15.

[78] S. M. Carroll, W. H. Press and E. L. Turner, Ann. Rev. Astron. Astrophys. 30 (1992) 499.

[79] J. P. Henry, Astrophys. J. 534 (2000) 565 [arXiv:astro-ph/0002365].

[80] B. Moore, T. Quinn, F. Governato, J. Stadel and G. Lake, Mon. Not. Roy. Astron. Soc. 310, 1147 (1999) [arXiv:astro-ph/9903164].

[81] H. J. Mo, Y. P. Jing and S. D. M. White, Mon. Not. Roy. Astron. Soc. 282, 1096 (1996) [arXiv:astro-ph/9602052]. 